\title{
Trivium
}

Revue franco-allemande de sciences humaines et sociales - Deutsch-französische Zeitschrift für Geistesund Sozialwissenschaften

$3 \mid 2009$

Droits subjectifs et droits de l'homme

\section{Die Proposition Égaliberté (»Gleichfreiheit«)}

\author{
Etienne Balibar
}

Traducteur : Achim Russer

\section{(2) OpenEdition}

\section{Journals}

Édition électronique

URL : http://journals.openedition.org/trivium/3337

ISSN : 1963-1820

\section{Éditeur}

Les éditions de la Maison des sciences de l'Homme

\section{Référence électronique}

Etienne Balibar, «Die Proposition Égaliberté (»Gleichfreiheit«)», Trivium [En ligne], 3 | 2009, mis en ligne le 24 avril 2009, consulté le 07 septembre 2020. URL : http://journals.openedition.org/trivium/3337

Ce document a été généré automatiquement le 7 septembre 2020

\section{(c) (i) () $\Theta$}

Les contenus des la revue Trivium sont mis à disposition selon les termes de la Licence Creative Commons Attribution - Pas d'Utilisation Commerciale - Pas de Modification 4.0 International. 


\title{
Die Proposition Égaliberté (»Gleichfreiheit«)
}

\author{
Etienne Balibar \\ Traduction : Achim Russer
}

\section{NOTE DE L'ÉDITEUR}

Wir danken Étienne Balibar für die freundliche Genehmigung, den vorliegenden Artikel in deutscher Übersetzung zu veröffentlichen.

\section{NOTE DE L'AUTEUR}

Der folgende Text stellt eine überarbeitete und erweiterte Fassung des Vortrags dar, den ich am 27. November 1989 im Rahmen der Conférences du Perroquet am Petit Odéon hielt und der erstmals in einer diesen Vorlesungen gewidmeten Reihe veröffentlicht wurde (Conférences du Perroquet, Nr. 22, November 1989). Eine zweite, gekürzte Fassung desselben Vortrags erschien unter dem Titel »Droits de l'homme et droits du citoyen: la dialectique moderne de l'égalité et de la liberté«, in: Balibar (1992).

Ich möchte einige Formulierungen anbieten, mit deren Hilfe wir uns in den Voraussetzungen einer für die achtziger Jahre typischen Diskussion besser zurechtfinden werden. Diese Diskussion, von Spezialisten geführt und zugleich auf die Öffentlichkeit zielend, war dadurch gekennzeichnet, dass sie das Thema der Beziehungen zwischen dem Politischen und dem "Sozialen" tendenziell durch das Thema der Beziehungen zwischen dem "Ethischen" und dem »Politischen" ersetzte und tiefgreifender vielleicht dadurch, dass sie dieses zweite Thema dem ersten wieder einbeschrieb. Dabei löste auf der »Rechten« wie auf der »Linken« die Frage nach der Citoyenneté, dem aktiven Bürgersein, die nach der Revolution ab. Sofern es nicht, grundlegender noch, um eine Neuformulierung des Problems der Revolution in Begriffen der Citoyenneté, also des staatsbürgerlichen Pflichtgefühls (Civisme) und 
Bürgersinns (Civilité) ging - sei es, indem eine Erneuerung der Citoyenneté (über die Anerkennung individueller Rechte hinaus) angestrebt wurde, sei es durch die Idee einer »neuen Citoyenneté«.

Es ist daher nicht erstaunlich, dass ein zentrales Thema der laufenden Debatten - von den Zweihundertjahrfeiern einmal abgesehen - Natur, Ablauf und historische Tragweite der Französischen Revolution betrifft, insbesondere ihren "Gründungstext«, die Déclaration des Droits de l'Homme et du Citoyen (»Erklärung der Menschen- und Bürgerrechte«) von 1789 - einen Text, dessen Bedeutung und universelle Geltung heute erneut befragt wird. Wenn auch ich dazu beitrage, die Aufmerksamkeit auf diesen Text zu lenken, bin ich mir der doppelten Gefahr - der Rückwärtsgewandtheit gegenüber den Herausforderungen der gegenwärtigen Geschichte und des Euro- oder gar Frankozentrismus - bewusst, den ein solches Herangehen an das politische Problem mit sich bringt. Aber selbst wenn die Frage der »Menschenrechte« nur eine Maske oder ein Köder wäre - was ich nicht glaube -, wäre es immerhin der Mühe wert, die Gründe für die Kluft zwischen dem Text von gestern oder vorgestern und einer aktuellen demokratischen Problematik zu ermessen. Und selbst wenn dieser Text nur der fiktiven Universalisierung einer besonderen Gesellschaft und Kultur entspräche - was ich auch nicht glaube -, wären auch dafür die Gründe zu ermitteln, und zwar mit erneutem Einsatz, anders als die geistigen Bewegungen und sozialkämpferischen Strömungen es taten, die unsere Idee von der »Revolution« im 19. und 20. Jahrhundert konfiguriert haben.

Im Folgenden möchte ich vier Aspekte dieser Frage mehr oder weniger eingehend erörtern.

Erstens: Wenn es zutrifft, dass der Text von 1789 (oder vielmehr seine aufeinanderfolgenden Formulierungen) für uns seit langem die Evidenz verloren hat, auf die er sich berief, wenn ferner zutrifft, dass zwischen den noch unlängst unauflöslich miteinander verbundenen Forderungen nach Freiheit und nach Gleichheit sich vielfach eine Kluft aufgetan hat - wie haben wir dann die Gründe dafür zu erklären?

Zweitens: Wie ist die Beziehung zwischen dem Text der Déclaration und der Spezifizität des revolutionären Ereignisses zu interpretieren? Ist die kollektive Praxis, die in diesem institutionellen Text ihren »Ausdruck" und ihre "Waffe» findet, unter der Kategorie eines Subjekts (Menschheit, "Zivilgesellschaft«, Volk, soziale Klasse) zu verbuchen oder eher als zeitbedingt, als Konstellation von Kräften? Eine Analyse des Charakters der Revolution von 1789-95 kann ich hier zwar nicht vorlegen, aber die Entscheidung für den zweiten Teil dieser Alternative wird mich veranlassen, einige Worte über die Neuartigkeit der Formulierungen der Déclaration im Verhältnis zu den klassischen Theorien vom »Naturrecht« $\mathrm{zu}$ verlieren, die gewöhnlich als ihre ideologische »Quelle« gelten.

Drittens: Um zum vermutlich Wesentlichen zu kommen, werde ich Aussagemodalität und -intentionalität jener Proposition prüfen, die, wie mir scheint, den Kern der Déclaration ausmacht und deren Logik zu verstehen erlaubt: die Proposition, die zunächst in ihrem Umfang und dann in ihrem Verständnis - „Freiheit « und "Gleichheit« miteinander gleichsetzt. Mich interessiert vor allem die Wahrheit dieser Proposition (die ich Égaliberté nenne) und damit der Riss durch das politische Feld, den sie verursacht; aber auch die Gründe ihrer Unbeständigkeit, die Entwicklungsformen der unaufhörlichen Trennung dessen, was als eine Einheit von Gegensätzen produziert 
worden war. Daraus folgt die Skizze eines Referenzsystems, einer »Topik«, mit deren Hilfe die unterschiedlichen Strategien zu klassifizieren und zu interpretieren sind - die theoretischen ebenso wie die politischen -, die sich während mindestens zwei Jahrhunderten (in Wirklichkeit sind wir nicht darüber hinaus) diesem Dilemma gestellt haben.

Viertens schließlich, wenn auch unvermeidlicherweise allzu oberflächlich, möchte ich folgendes Problem zumindest anreißen: Wenn die revolutionäre Proposition, die Freiheit mit Gleichheit identifiziert, tatsächlich die unumgängliche und in gewissem Sinn irreversible Äußerung einer politischen Wahrheit darstellt, wenn weiterhin zutrifft, dass die Einschreibung dieser Wahrheit in die Geschichte selbst, die sie produziert hat, sich umgehend durch ihre Unbeständigkeit und in gewissem Sinn durch Ihren Verfall kennzeichnet, wenn schließlich wahr ist, dass ihre Rückkehr in die politische Aktualität zumindest die Forderung nach einer Wiedereinschreibung oder einer neuen praktischen Einschreibung indiziert, dann stellt sich die Frage, unter welchen Bedingungen diese am Ende des 20. Jahrhunderts denkbar wäre. Eine solche Frage muss weitgehend offen und wohl auch aporetisch bleiben. Zumindest wird es jedoch möglich sein, die Gründe dafür zu erhellen, indem negativ benannt wird, welche Widersprüche der modernen Politik stillschweigend übergangen, grundlegender noch: in der um die Déclaration konstruierten Topik verdrängt wurden.

Erstens also: Wie stellt die Aktualität der revolutionären Aussagen sich dar? Ich sagte es gerade: in der paradoxen Form einer scheinbar unreduzierbaren Kluft zwischen Begriffen oder Werten, die doch als gleichermaßen notwendig empfunden werden. Zwar bezeugt die Interdependenz von Gleichheit und Freiheit sich a contrario in Gestalt der periodischen Wiederkehr von Autoritätsideologien, die behaupten, dass das Leben in Gesellschaft oder die menschliche Natur eine Hierarchisierung der individuellen oder auch kollektiven Ungleichheit und deren Valorisierung erforderlich machten. Aber diese Permanenz der zu Anfang des 19. Jahrhunderts durch das konterrevolutionäre Denken inaugurierten "Kritik der Menschenrechte« bringt keinerlei Kohärenz hervor. Nicht nur der zeitgenössische Liberalismus behauptet, dass "Freiheit" und "Gleichheit" sich außerhalb sehr enger (rechtlicher) Grenzen gegenseitig ausschließen: Diese Überzeugung wird auch vom Sozialismus oder allgemeiner vom sozialen Fortschrittsglauben unterschiedlicher "Minderheiten " ${ }^{1}$ weitgehend geteilt, und dies zu einem Zeitpunkt, da sich praktisch zeigt, dass die Forderungen nach Freiheit und Gleichheit einander gegenseitig bedingen - dies machen die Kämpfe um die Demokratie in den ehemaligen "sozialistischen Ländern" ebenso deutlich wie die antirassistischen Bewegungen Westeuropas oder die Kämpfe der Schwarzen in Südafrika.

Diesen tiefen Widerspruch nähren mehrere selten in Frage gestellte, augenscheinliche Gewissheiten: insbesondere die, der zufolge die Gleichheit (generell wird präzisiert: die »reale Gleichheit«) wesentlich in der wirtschaftlichen und sozialen Sphäre - ein von Natur aus elastischer Begriff, der heute tendenziell auch das "Kulturelle« einschließt zu Hause ist, während die Freiheit vor allem der juristisch-politischen und institutionellen Sphäre angehört. Gleichzeitig aber gilt als gewiss, worin Liberalismus und Sozialismus schließlich übereingekommen sind (selbst wenn sie entgegengesetzte Folgen daraus ableiten): dass die Gleichheit durch den Eingriff des Staats zu verwirklichen ist, weil sie wesentlich mit Verteilung oder Umverteilung zu tun hat, während der Schutz der Freiheit an die Begrenzung dieses Eingriffs, ja an die permanente Verteidigung gegen seine "unbeabsichtigten Folgen", gebunden ist. ${ }^{2}$ 
Ebendieser omnipräsente, aber unkritische Bezug auf den als Block konzipierten Staat reproduziert unaufhörlich sowohl den Unterschied zwischen "formalem " und »realem» (oder "substanziellem«) Recht als auch die Vorstellung von Gleichheit als ausschließlich kollektivem Ziel, während die Freiheit (jedenfalls die »Freiheit der modernen Welt«) wesentlich individuelle Freiheit sein soll, und das selbst in der Sphäre der öffentlichen Freiheiten (die also wesentlich als öffentliche Garantien privater Freiheiten vorzustellen wären).

Von hier aus ist es nicht weit zum Grundparadox, der Kluft, die sich zwischen dem Diskurs der »Menschenrechte« und dem der »Bürgerrechte« auftut - und dies zu einem Zeitpunkt, da eine Moralisierung oder Neubegründung des politischen Lebens sich auf die Ethik beruft. Der Diskurs der Menschenrechte (der sich vor allem als Verteidigung, weit weniger als Eroberung dieser Rechte gibt) deckt heute ein breites Spektrum, das sich von der Gewissensfreiheit oder persönlichen Sicherheit bis zur Forderung nach dem Recht auf Leben oder dem Recht der Völker auf Selbstbestimmung erstreckt. Er bleibt jedoch grundverschieden vom Diskurs der Bürgerrechte, der seinerseits zwischen der Forderung nach einer Erweiterung der politischen Sphäre auf neue Bereiche (zum Beispiel die Ökologie) und der nach einer Aufwertung der klassischen Politik - des Synonyms einer kollektiven Institution zur Beratung und Entscheidung - gegen das Vordringen des Ökonomismus und Technokratismus oszilliert. Es scheint äußerst schwierig und vielleicht immer schwieriger, die für die revolutionären Formulierungen von 1789 typische Gleichsetzung zu vertreten, auf die ich zurückkommen werde: die von »Mensch" und »Citoyen«, die unter anderem den Gedanken zur Folge hatte, dass die Emanzipation der Unterdrückten nur ihre eigene Sache sein kann. Als wäre der Mensch nichts anderes als das, was übrig bleibt, wenn man vom Staatsbürger absieht. Es fehlt nicht viel, und die Gleichsetzung von Mensch und Citoyen würde vom allgemeinen Konsens als Weg zum Totalitarismus verschrien, zu dem, was oft auch als Imperialismus des "Alles ist politisch" denunziert wird: Aber die Kehrseite dieses Konsenses ist, dass die Verkündung der Menschenrechte, so natürlich und universell die Forderung nach ihnen auch sein mag, im Wesentlichen ein Ideal darstellt. Dies ist bei näherem Hinsehen eine exakte Verkehrung des "performativen« Textes von 1789, der ihre brennende soziale Aktualität deklariert und die Notwendigkeit und Möglichkeit ihrer Inkraftsetzung, ihre Materialisierung in einer "Verfassung" postuliert. ${ }^{3}$

Man kann und muss sich nach den Gründen für diese Kluft fragen, die flagrant erscheint zu einem Zeitpunkt, da der Bezug auf den juristischen Universalismus erneut aktuell wird. Mehrere Erklärungen sind möglich, die wir gut kennen. Die eine beruft sich auf die menschliche Natur: Zwischen »Menschenrechten« und »Bürgerrechten« liege dieselbe Kluft wie zwischen der wesentlichen, theoretischen Güte der menschlichen Natur, ohne die eine wahre Gemeinschaft undenkbar wäre, und der praktischen Bosheit der empirischen Individuen, dieser Opfer ihrer Leidenschaften, Interessen, Lebensbedingungen. Homo homini deus, homo homini lupus. An dem unwahrscheinlichen Punkt, an dem diese Gegensätze sich ausgleichen, finden wir das Recht, das J.D. Bredin kürzlich als »Kunst der Lösung unlösbarer Fragen« zu definieren vorschlug ${ }^{4}$, was im Grunde auf Kants »ungesellige Geselligkeit« hinausläuft. Eine überaus beliebte, ebenso banale Erklärung ist die historizistische: Die Zeit ging vorüber, die materiellen und kulturellen Bedingungen, unter denen die Evidenz der Formulierungen von 1789 ins Auge sprang, sind nicht mehr. Wir sind gewiss nicht mehr die "Menschen" des 18. Jahrhunderts, und ob wir noch die "Bürger» des 
19. Jahrhunderts sind, steht dahin. In gewisser Hinsicht sind wir mehr als das (zum Beispiel leben wir in einer Welt globaler Kommunikation und Kultur, was die Staatsangehörigkeit, diesen unüberschreitbaren Horizont der revolutionären Periode, relativiert), in anderer Hinsicht sind wir weniger, insofern uns nicht so sehr die Klassenzugehörigkeit als vielmehr der Status kennzeichnet, den unsere "differenzierten« Gesellschaften ihren Mitgliedern zuweisen. Es ist nicht unmöglich, beide Erklärungstypen miteinander zu kombinieren: so, wenn wir den ursprünglichen Utopismus der Menschenrechte hervorheben und postulieren, dass ihre Verkündung von vornherein - da sie der politischen und sozialen Moderne das Mittel lieferte, sich gegen die hierarchischen Sozialordnungen der Vergangenheit und ihre eigene, vor allem theologisch geprägte Imagination zu behaupten - selbst nur die Funktion hatte, ein Ideal zu formulieren, das heißt ein neues Imaginäres zu kristallisieren.

Ich gebe einem anderen, dialektischeren oder, wenn man so will, immanenteren Erklärungsmodus den Vorzug: dem, dass die »Gründungsformulierungen« gerade auf Grund ihrer Schlichtheit und revolutionären Radikalität von vornherein einen Widerspruch in sich bergen, der ihnen verbietet, sich in eine stabile Ordnung umzusetzen. Oder besser noch: dass der Widerspruch auf höherer Ebene in der Instabilität der Beziehung zwischen dem aporetischen Charakter der Formulierungen ${ }^{5}$ und dem konfliktgeladenen Charakter der Umstände besteht, aus denen sie hervorgehen und die ihnen als Bezugsgröße dienen. So dass jedem sich auf die genuine Wahrheit des Texts der Menschen- und Bürgerrechte gründenden Versuch zu seiner Reaktivierung die Folgen der Entwicklung seiner eigenen inneren Spannungen entgegenstehen. Dieser Weg scheint mir der am stärksten Erfolg versprechende; er lässt sich auf verschiedene Weise beschreiten, was ich nicht im Einzelnen erörtern möchte. Hier erlangt vor allem die Interpretation der Entwicklung der Déclaration innerhalb des revolutionären Prozesses von 1789-95 eine entscheidende Bedeutung, namentlich in der Konfrontation des ursprünglichen Textes mit den mehr oder weniger gescheiterten, aber nicht folgenlos gebliebenen Neufassungen von 1793 (die »jakobinische«) und von 1795 (die »thermidorianische«). Denn diese Entwicklung mit ihrem charakteristischen Schwanken zwischen zwei "Lesarten" der Beziehung Mensch / Citoyen, einer plebejischen und einer bürgerlichen, stützt sich auf der Revolution selbst innewohnende antagonistische Kräfte und ist bereits aufschlussreich für die Widersprüche, die seit Beginn am Werke sind.

In seinem bemerkenswerten Buch La Révolution des Droits de l'Homme ${ }^{6}$ hat Marcel Gauchet sich vom neoliberalen, um nicht zu sagen konservativen Gesichtspunkt aus auf die Suche nach den Gründen gemacht, aus denen die Revolution für uns »beendet « ist (aber auch nach jenen, die dieses Ende so lange »hinausgezögert« haben). Er hat von Text zu Text verfolgt, was ihn die Entfaltung einer grundlegenden Aporie dünkt: dass es sich bei der Déclaration von 1789 im Kern um die Formulierung eines absoluten Begriffs von nationaler Souveränität handelt, um die mimetische Umkehrung der Souveränität des Monarchen, der sich die Déclaration widersetzt, um die Volksvertretung zu legitimieren. Dem (zumindest theoretisch) »einen und unteilbaren« Willen des absoluten Monarchen muss der Konvent die ebenfalls »eine und unteilbare« volonté générale entgegensetzen, auch sie ausschließlicher Hoheitsträger, aber letzten Endes einzig auf den Individuen beruhend, aus denen die Nation besteht. Ein solcher Begriff, so Gauchet, ist dazu verurteilt, zwischen Allem oder Nichts, zwischen direkter Demokratie und revolutionärer Diktatur zu schwanken: Im Unterschied zu den partiellen, aber eindeutig festgelegten Freiheiten des amerikanischen »Modells« der Bill 
of Rights erweist er sich - ob beim Austarieren der Befugnisse zwischen Legislative und Exekutive oder zwischen Prärogativen des Staats und Unabhängigkeit der Individuen als unvereinbar mit der pragmatischen Einrichtung eines rechtlichen Rahmens für die moderne Politik. Deswegen scheitert die Revolution zunächst, und erst in einem ganz anderen Kontext - nach einem Jahrhundert politischer Konfrontationen und Regimekrisen - erhalten ihre symbolischen Formulierungen die Funktion eines mehr oder weniger Konsens stiftenden, regulativen Ideals.

Symmetrisch dazu hat Florence Gauthier in einer Reihe von Beiträgen ${ }^{7}$, in denen sie die Tradition des revolutionären Idealismus (die sich von Robespierre und Fichte über den jungen Marx bis hin zu Ernst Bloch erstreckt) aufgreift und erneuert, zu zeigen versucht, dass zwischen der jakobinischen und der thermidorianischen Phase des Konvents ein Bruch eingetreten ist. Die auf den Primat der Freiheit und das Streben nach ihrer Universalisierung ausgerichteten Formulierungen von 1789 stehen in kontinuierlichem Zusammenhang mit denen von 1793, die den latenten Egalitarismus dieser Konzeption als universelle Gegenseitigkeit oder universelle gegenseitige Anerkennung der Freiheiten einschließlich der Grundfreiheit zu leben (des »Rechts auf Leben« mit all seinen ökonomischen Konsequenzen) entwickeln. Sie gehen aus der klassischen, im Wesentlichen Lockeschen Vorstellung einer Erklärung des Naturrechts hervor, die den Zusammenschluss oder die Citoyenneté begründet und der politischen Sphäre und der Rolle des Staats von der menschlichen Natur her Schranken setzt. In der thermidorianischen Déclaration von 1795 hingegen, in deren Mittelpunkt der unantastbare Charakter des Eigentums und die Reziprozität von »Rechten" und "Pflichten« stehen, wird die universelle natürliche Grundlage der Citoyenneté durch eine "soziale« Begründung ersetzt: ein Bruch, ja eine Umkehrung. Darin findet natürlich die konterrevolutionäre Reaktion auf die sozialen Konflikte ihren Ausdruck, insbesondere die Reaktion auf die Art und Weise, in der die plebejischen Elemente der Revolution den Universalismus der Menschenrechte ständig politisch eingesetzt haben, und zwar gegen die praktischen Einschränkungen durch deren Verfasser: Unterscheidung von "aktiver" und "passiver" Citoyenneté auf Basis des Zensuswahlrechts, Ausschluss der "faktischen Gleichheit« aus dem Bereich der Naturrechte.

Ich mache mir keine dieser beiden Möglichkeiten zu Eigen, den immanenten Widerspruch des revolutionären Augenblicks zu interpretieren. Beide scheinen mir aus ganz unterschiedlichen Gründen dessen Spezifizität zu verfehlen, ohne die weder die unmittelbaren Folgen der Formulierungen noch ihre späteren, bis auf heutige Tage sich erstreckenden Auswirkungen zu verstehen sind: die Spezifizität des Textes der Déclaration, die Spezifizität der revolutionären Umstände selbst. Leider kann ich dies hier nicht im Einzelnen darlegen. Schematisch gesprochen glaube ich weder, dass das 1789 unter ganz bestimmten Umständen geschmiedete Konzept der "Souveränität der Nation« die Umkehr der monarchischen Souveränität im Rahmen einer grundsätzlich weiter bestehenden Kontinuität darstellt, gewissermaßen die Ersetzung einer Transzendenz durch eine andere; noch, dass der Bezug auf den Menschen und auf die Universalität seiner die Bürgerrechte »begründenden« Natur hier auf den Durchschnittstenor der ideologischen Quellen zurückführbar ist, die sich allgemein als klassisches "Naturrecht « bezeichnen lassen.

Was die Souveränität angeht, so besteht die revolutionäre Innovation, wie ich andernorts zu zeigen versucht habe ${ }^{8}$, gerade darin, ihr herkömmliches Konzept durch 
die höchst paradoxe These einer egalitären Souveränität umzustürzen - praktisch eine contradictio in adiecto, aber die einzige Möglichkeit, aller Transzendenz radikal den Laufpass zu geben und die politische und soziale Ordnung dem Element der Immanenz, der Selbstkonstitution eines Volkes einzubeschreiben. Daher aber auch die sofortige Entwicklung einer ganzen Reihe von Widersprüchen, die daraus hervorgehen, dass die sogenannte "Zivilgesellschaft» und erst recht der Staat ganz und gar auf Hierarchien oder Abhängigkeiten aufbauen, die der Souveränität gleichgültig gegenüberstehen und doch zugleich zu ihrer Institutionalisierung wesentlich sind, während die Gesellschaft oder moderne Polis nicht mehr über das der antiken Polis eigene »Mittel« verfügt, diese Widersprüche zu neutralisieren oder aus der öffentlichen Sphäre zu verdrängen: die strenge Trennung zwischen oikos und polis.

Was das deklarierte Naturrecht angeht, so halte ich den revolutionären Moment der Déclaration und sein ungebrochenes Fortwirken in den soziopolitischen Auseinandersetzungen tatsächlich für wesentlich. Anders gesagt, ich zweifle nicht daran, dass die Materialität dieses Aktes der Punkt war, an dem die im Anschluss daran sofort aufkommenden Forderungen nach Einfügung des Frauenrechts, des Rechts der Arbeiter, der kolonisierten "Rassen « in die Citoyenneté sich verankerten und auf den sie sich beriefen. Ich glaube aber keineswegs, dass er als Endpunkt, Vollendung oder schlicht als Radikalisierung des klassischen Naturrechts, ob Lockescher oder selbst Rousseauscher Obödienz, in dessen Kontinuität steht. ${ }^{9}$ Welches Bewusstsein auch immer die Verfasser beim Ringen mit ihren eigenen, aus der Zeit des Ancien Régime stammenden geistigen Voraussetzungen von sich selbst gehabt haben mögen: Historisch und epistemologisch hat der Kern der Déclaration des Droits de l'Homme et du Citoyen nichts mit präexistenten Ideologien zu tun, er verlässt den Rahmen der Theorien über die "menschliche Natur" als Grundlage oder Gewähr einer Rechtsordnung, die vom 16. bis zum 18. Jahrhundert die Alternative zu den Theorien vom "göttlichen Recht" bildete und den Opponenten der Monarchie die Basis ihrer Argumentation lieferte. Greift er ihre Terminologie - teilweise - auf, so lediglich, um ihre Logik außer Kraft zu setzen. Unmittelbar löst er nicht den Triumph, sondern die Krise des klassischen Naturrechts aus, öffnet er den Raum für ein neues ideologisches Feld, in dem die politisch-philosophischen Ideologien des 19. Jahrhunderts - ob Liberalismus, Sozialismus oder Konservatismus, ob epistemologisch dem Positivismus oder dem Historizismus, dem Idealismus oder dem politischen Pragmatismus verpflichtet - sich ansiedeln werden.

Das klassische Naturrecht ist durch die extreme Unterschiedlichkeit seiner Konzeptionen der menschlichen Natur und seiner Vorstellungen vom ursprünglichen Zustandekommen der Gesellschaft gekennzeichnet, was ebenso vielen Reformstrategien für die politische Institution entspricht. Die Formulierungen von 1789 hingegen, den Debatten der dreifach - durch die eigenen Interessen, durch den offenen, noch unentschiedenen Konflikt mit der Monarchie und durch die Angst vor Volksaufständen - bedrängten "Nationalvertretung" mittels eines wahren Gewaltstreichs entsprungen, kennzeichnet eine bemerkenswerte Schlichtheit (die ich andernorts eine Rechtstatsache genannt habe) ${ }^{10}$, von der wir sehen werden, dass sie im Grunde rein negativ ist, dass sie die Problematik der Ursprünge und der Modalitäten des Zusammenschlusses umgeht. ${ }^{11}$ Bemerkenswert ist vor allem, dass der Begriff "Vertrag" hier fehlt. ${ }^{12}$ Aber selbstverständlich »entspricht « die Komplexität und Heterogenität der Theorien des klassischen Naturrechts, ob kontraktualistisch oder antikontraktualistisch, ob etatistisch oder ökonomistisch, der relativen Homogenität 
einer aufsteigenden sozialen Klasse, die als Bürgertum bezeichnet werden kann, während die vereinheitlichende Schlichtheit der Déclaration im Feld der Ideen oder vielmehr der Worte - Worte, die sich der Kontrolle ihrer Urheber sofort entziehen der realen gesellschaftlichen Komplexität der Französischen Revolution entspricht: der Tatsache, dass sie von vornherein bereits keine »bürgerliche Revolution« mehr ist, sondern eine vom Bürgertum und dem Volk, oder genauer den nicht bürgerlichen und noch weniger kapitalistischen Massen, in Bündnis und Konfrontation gemeinsam durchgeführte Revolution. Eine Revolution, die sogleich mit ihrer eigenen internen Opposition ringt, ohne die sie nicht wäre, und die unablässig auf der Suche nach der Einheit ihrer Gegensätze ist.

Kommen wir also zum Kern der revolutionären Formulierungen. Er besteht, wie mir scheint, in einer doppelten Gleichsetzung, von denen die eine die andere erklärt und ihr ihren Inhalt verleiht (aber dieser Inhalt bleibt, wie wir noch sehen werden, seltsam unbestimmt).

Erste Gleichsetzung: die von Mensch und Citoyen. Hier müssen wir uns für eine Lesart entscheiden, denn eine lange und quasi-offizielle nationale und internationale Tradition interpretiert den Inhalt der ursprünglichen siebzehn Artikel als Ausdruck einer Unterscheidung der (universellen, unveräußerlichen, von jeder sozialen Institution unabhängigen, also virtuellen usw.) »Menschenrechte" von den (positiven, instituierten, eingeschränkten, aber realen) "Staatsbürgerrechten" - eine Unterscheidung, die dazu führt, dass die zweiten auf den ersten gründen..$^{13}$ Wenn es etwas zu »begründen" gilt, ist die Grundlage in der Tat von dem zu unterscheiden, was auf ihr beruht. Die Frage ist aber eben die, ob wir es im Text selbst eigentlich mit einer "Grundlegung" zu tun haben. ${ }^{14}$ Gewiss beinhaltet die Dualität der Begriffe »Mensch" und "Citoyen« die Möglichkeit ihrer Dissoziierung - wir werden die Auswirkungen sehen. Im gegebenen Kontext jedoch ist sie anders zu interpretieren. Eine nochmalige Lektüre der Déclaration erweist unzweideutig: Zwischen "Menschenrechten« und "Staatsbürgerrechten« besteht in Wirklichkeit keinerlei Kluft, keinerlei inhaltlicher Unterschied - es sind genau dieselben. Folglich gibt es auch keinen Unterschied zwischen Mensch und Citoyen, zumindest insofern, als sie praktisch durch ihre Rechte definiert werden - durch die Natur und den Umfang der Rechte, deren Inhaber sie sind: Und genau darum geht es in der Déclaration. Ich erinnere daran, dass als "natürliche und unantastbare Rechte des Menschen" Freiheit, Eigentum, Sicherheit und Widerstand gegen Unterdrückung aufgezählt werden (Art. 2), das heißt genau die Rechte, von denen die Déclaration in ihrem weiteren Verlauf zeigt, dass die soziale Verfassung ihre Rechtsorganisation darstellt.

Welche Probleme stellen sich auf dieser Ebene? Zunächst einmal das angeführte Recht auf Widerstand gegen Unterdrückung. Das mindeste, was man sagen kann, ist, dass es später nicht gerade ausdrücklich verankert wurde. Zugleich liegt aber auf der Hand, dass es das Korrelat der Freiheit ist, die Gewähr dafür, dass sie tatsächlich existiert frei sein heißt, jedem Zwang widerstehen zu können, der die Freiheit zerstört - und eine sprachliche Spur des revolutionären Kampfs, der diese Freiheit errang und durchsetzte. ${ }^{15}$ Ferner das scheinbare Fehlen der Gleichheit, ein Hinweis auf innere Schwankungen, von denen noch zu sprechen sein wird. Diesen Eindruck gilt es jedoch sogleich richtigzustellen. Artikel 1 erklärt: "Die Menschen werden frei und gleich an Rechten geboren und bleiben es [...]«, Artikel 6: „Das Gesetz ist Ausdruck des allgemeinen Willens. Alle Bürger haben das Recht, [daran] mitzuwirken [...] Es muss 
dasselbe für alle sein [...] Da alle Bürger vor ihm gleich sind, gilt es für alle gleichermaßen [...]«. Diese Formulierungen gleichen das »Fehlen« der Gleichheit in Artikel 2 nicht nur aus, sie kehren seine Bedeutung um, indem sie die Gleichheit zum Prinzip oder Grundrecht machen, das alle anderen erst zu einer Einheit verknüpft.

Die Art und Weise, wie die Gleichheit in der Déclaration behandelt wird, stellt gerade die stärkste und präziseste Gleichsetzung zwischen dem Menschen und dem Citoyen dar. Dies wird ihr bald schon zum Vorwurf gemacht, und es wird sehr rasch dazu führen, auf die eine oder andere Weise Mensch und Citoyen, "Menschenrechte" und "Bürgerrechte« zu dissoziieren, während wir hier, im Moment der Revolution, mit dem der Akt des Formulierens (die Déclaration) unlösbar verbunden ist, ihr Koinzidieren bekräftigt finden. Nicht nur etabliert die Déclaration keinerlei "menschliche Natur" oberhalb der Gesellschaft, der politischen Ordnung, als eine Art Fundament oder äußere Garantie, womit sie Theorien über die menschliche Natur wie über das theologische Übernatürliche umgeht: Sie identifiziert Menschenrechte mit politischen Rechten, den individuellen oder kollektiven Menschen mit der politischen Gesellschaft. Im Folgenden werde ich diesen Begriff der Menschenrechte als politischer Rechte und umgekehrt, der politischen Rechte als Menschenrechte ausführen und zeigen, dass er bis zur Idee der Menschenrechte auf Politik reicht: soweit, alle menschliche Tätigkeit politisch, nämlich in die Perspektive einer Befreiung und Gleichstellung zu instituieren. Aber zunächst einmal wird es nicht unnütz sein darüber nachzudenken, was diesen Begriff so radikal von den - eben "naturalistischen« - Formulierungen der antiken Tradition unterscheidet. Die Gleichsetzung von Mensch und Citoyen aus dem Jahr 1789 ist keine Reprise des zoon politikon, sondern eher eine Umprägung dieses Begriffs, die die Möglichkeit eines Einschnitts voraussetzt. Die Idee des zoon politikon basiert nämlich sofern sie den Institutionen der griechischen oder römischen »Polis« überhaupt entspricht - nicht auf der Gleichsetzung von Gleichheit und Freiheit, sondern auf einer ganz anderen These: auf der Gleichheit in den Grenzen der Freiheit, wobei letztere als sozialer Status betrachtet wird, der je nachdem auf einer Tradition, einer Verfassung oder einer natürlichen Qualität der Individuen beruht (die für sie selbst und für andere aus ihnen »Herren« macht). Die Gleichheit ist hier nur eine Konsequenz, ein Attribut der Freiheit, das Verhältnis beider Begriffe zueinander ist nicht umkehrbar. Daher die seltsame Begrenztheit des Begriffs Staatsbürgerschaft - oder wenigstens das, was dem modernen Leser retrospektiv so erscheinen muss - bis in die Texte hinein, die seine demokratischen Potenzialitäten am tiefsten ausloten, manche Passagen der Politik des Aristoteles zum Beispiel. Dieser »definiert« Staatsbürgerschaft durch die abwechselnde Ausübung von Funktionen des Herrschens und Gehorchens (archein / archesthai), also durch eine starke Form verallgemeinerter Reziprozität der freien, männlichen und erwachsenen Individuen (die auch die Basis ihrer philia ist: ein Oberbegriff von "sozialem Band«). Dieser Reziprozität entspringt eine anthropologische, ja kosmologische Position des "Staatsbürgers" zwischen zwei Grenzen, die nach unten und nach oben gleichzeitig Grenzen der Politik bezeichnen: der Untermensch in seinen unterschiedlichen Gestalten (Frau, Sklave, Kind), der Übermensch als Weiser, Gott oder Held. Aber dort, wo wir retrospektiv nur die widersprüchliche Verbindung eines Ansatzes zur Universalität mit einer willkürlichen Einschränkung sehen, herrscht in Wirklichkeit eine andere Logik, in der »Freiheit» einen Status darstellt, eine persönliche Rolle, und »Gleichheit« eine mit diesem Status korrelierende Funktion, ein ihm entsprechendes Recht. ${ }^{16}$ 
Umgekehrt wäre es genau so irrig, unter dem Vorwand historischen Bewusstseins den klassischen, aus dem Liberalismus hervorgegangenen Gegensatz zu übernehmen und zu meinen, im Gegensatz zur griechischen (und mehr noch zur römischen) Einheit von Sozialem und Politischem habe die Déclaration von 1789 deren Trennung - oder deren "bürgerliche« Trennung - instituiert, die ihrerseits auf der Unterscheidung zwischen öffentlicher und privater Sphäre beruhe. Dass Marx sich diese Lesart seiner Zeitgenossen in einer seiner berühmten Frühschriften zu Eigen machte ${ }^{17}$, hindert nicht, dass sie zum Wortlaut und zum materiellen Gehalt jener Formulierungen in fundamentalem Widerspruch steht. Der Mensch der Déclaration ist nicht das "private Individuum « im Gegensatz zum Bürger als Mitglied des Staates. Er selbst ist der Citoyen, und diese Feststellung wirft umgekehrt die Frage auf, wie es eigentlich kommt, dass der Staatsbegriff sich derart problematisch ausnimmt - und das in einem revolutionären Text, der als Grundlage einer Verfassung dienen will und dessen Bestimmung (zumindest in den Augen seiner Verfasser) die Errichtung eines neuen Staats ist. Die Antwort auf diese Frage kann nur aus der Prüfung der subversiven Wirkungen eines radikal neuen Gedankens hervorgehen, der eben die Beziehung von Gleichheit und Freiheit zum Gegenstand hat und universelle Geltung beansprucht.

Worin besteht dieser Gedanke? In nichts weniger als der Gleichsetzung beider Begriffe. Das ist die außerordentliche Neuerung, und sie ist zugleich die Wurzel aller Schwierigkeiten, der Kern des Widerspruchs. Buchstäblich genommen sagt die Déclaration tatsächlich aus, dass die Gleichheit der Freiheit gleich, mit Freiheit identisch ist, und umgekehrt. Jede der beiden ist das exakte »Maß« der anderen. Dafür schlage ich den bewusst barocken Begriff Égaliberté (»Gleichfreiheit«) vor, eine im Französischen »unmögliche« Wortzusammensetzung und doch nur als französisches Wortspiel möglich ${ }^{18}$, das für sich genommen bereits die zentrale Aussage enthält. Denn es benennt die Bedingungen, unter denen der Mensch Citoyen ist (und ganz und gar Citoyen) und den Grund der Gleichsetzung. Hinter oder vielmehr in der Gleichung Mensch = Citoyen steckt die Proposition Égaliberté: als Grund ihrer Universalität.

Diese Proposition hat den Status einer Evidenz (self evident truth, wie die Amerikaner sagen). Oder vielmehr den einer Gewissheit, das heißt: Ihre Wahrheit kann nicht in Zweifel gezogen werden. Wie kommt es aber dazu, dass dies trotzdem und sogar ständig geschieht, wiewohl in Negierungsformen, die unaufhörlich deren Dringlichkeit einräumen, ihre Unwiderruflichkeit nachweisen?

Das kann nicht einfach daran liegen, dass wir hier zwei Wörter haben. Ihre formale Unterscheidung ist natürlich Voraussetzung dafür, ihre Bedeutungen gleichzusetzen. Besser gesagt: Damit Freiheit und Gleichheit als identisch gedacht werden können, muss eine ursprüngliche Differenz reduziert werden, die sich in den relativ getrennten Geschichten der Wörter »Freiheit« und »Gleichheit« vor dem Text von 1789 eingelagert hat, bis sie sich in einer Weise begegnen, die mit einem Schlag das gesamte Bild verändert. In anderer Hinsicht ist dies einfach Ausdruck dessen, dass die Revolutionäre von 1789 gegen zwei Gegner und zwei Prinzipien gleichzeitig kämpften: gegen den Absolutismus, der als Negation der Freiheit erscheint (in den ständigen königlichen Willkürmaßnahmen), und gegen die Privilegien, die als Negation der Gleichheit erscheinen (im ständigen "Recht des Stärkeren«, das heißt der "Standespersonen«). Gegen Monarchie und Aristokratie, deren politisch-soziale Einheit von ihnen sofort in dem Begriff »Ancien Régime« zusammengedacht wird, diesem Amalgam, mit dem das konterrevolutionäre Denken bis heute hadert, namentlich indem es die Revolution in 
eine "Revolution der Freiheit» und eine »Revolution der Gleichheit» auseinanderdividiert. Aber die Revolution von 1789 ist gleichzeitig das eine und das andere (und eben deswegen ist sie die Revolution, bewirkt sie augenblicklich eine irreversible Mutation der Bedeutung dieses Worts): Sie wendet sich gegen »Tyrannei« wie gegen "Ungerechtigkeit« (gegen eine Gleichheit in der Unterwerfung wie gegen eine mit Privilegien identifizierte Freiheit); sie zeigt, dass eine gerechte Tyrannei (eine aufgeklärte Despotie ...) so unmöglich ist wie eine demokratische Ungerechtigkeit.

Aber was tiefgreifender noch der Anerkennung dieser radikalen These im Wege steht, ist das, was man die platonische Lektüre der Texte nennen muss: Man sieht Gleichheit und Freiheit als Ideen oder Gattungen, dem Gesetz von Eigenem und Fremdem unterworfen. Man sucht die gemeinsame Natur von Gleichheit und Freiheit, sucht nach dem Punkt, durch den sie schließlich ihr gemeinsames »Wesen« enthüllen könnten (und fast ist man versucht, mit einer Tautologie $\mathrm{zu}$ antworten: Diese Natur, dieses »Wesen«, in einem Wort: diese Grundlage ist der Mensch oder das Subjekt). Es gibt auch einen anderen Grund: Man ahnt, dass man dieser Identität einen »empirischen Inhalt«, eine positive »Referenz« nur geben könnte, wenn sich angeben ließe, welche Freiheit, welche Gleichheit identisch sind, oder vielmehr - denn offenkundig setzt der Text der Déclaration sie universell, in allen ihren Formen miteinander gleich - in welchen Grenzen oder unter welchen Bedingungen sie miteinander identisch sind. Kurz, wir stoßen hier auf eine verblüffende Unbestimmtheit.

In Wirklichkeit handelt es sich um zwei miteinander verbundene, aber unterschiedliche Fragen. Für die erste fällt die Antwort leicht, und doch ist sie folgenschwer, weil sie sich auf nichts weniger als den Wahrheitsstatus der Proposition Égaliberté auswirkt. Was die zweite Frage betrifft, so ist sie praktisch nicht zu beantworten, oder vielmehr: Sie ist dazu bestimmt, durch ihren Widerspruch selbst unabsehbar vertagt zu werden, was gewiss nicht weniger schwer wiegt, da es bei ihr schlicht um die Anwendung, um den Übergang von der »Theorie« zur »Praxis« einer Proposition geht, die aus der (revolutionären) Praxis selbst hervorgegangen ist.

Greifen wir zunächst die Frage der Natur auf. Meine Position ist eindeutig: Die der Proposition der Égaliberté zugrunde liegende Gleichung (Gleichheit = Freiheit) ist nicht essentialistisch. Was ihr zugrunde liegt, ist nicht die intuitive Entdeckung oder Offenbarung einer Identität der Ideen Gleichheit und Freiheit, sei es auch nur, weil diese aus ihrer revolutionären Gleichsetzung vollkommen verwandelt hervorgehen. Es ist vielmehr die historische Entdeckung, die ganz zu Recht experimentell genannt werden kann, dass ihr Umfang notwendig identisch ist. Um es noch deutlicher zu sagen: Die Situationen, in denen eine von beiden fehlt, sind zwangsläufig dieselben, in denen auch die andere fehlt. Noch anders gesagt: Die (faktischen) historischen Voraussetzungen der Freiheit sind genau dieselben wie die (faktischen) historischen Voraussetzungen der Gleichheit.

Ich behaupte, dass die so verstandene Proposition Égaliberté durchaus eine Wahrheit ist, und zwar eine irreversible Wahrheit, entdeckt im revolutionären Kampf und durch ihn - eben die universell wahre Proposition, auf die die verschiedenen »Kräfte«, aus denen sich das revolutionäre Feld zusammensetzte, sich einigen mussten. Die historischen Auswirkungen dieser Proposition wiederum können, so widersprüchlich sie sein mögen, ihrerseits nicht anders begriffen werden denn als Auswirkungen einer Wahrheit oder Wahrheitseffekte. Was nicht besagt, dass sie niemals vergessen, niemals zurückgewiesen worden wären. 
Sie werden mir sagen: Welchen Beweis gibt es dafür? Bei einer universellen Wahrheit in diesem Sinn (universell a posteriori oder besser: historisch universell) kann der Beweis nur negativ geführt werden (er kann nur den Status eines "Gegenbeweises« haben, eines elenchos, wie Aristoteles gesagt hätte), aber dies zu jeder Zeit und in allen möglichen Situationen. Wenn absolut wahr ist, dass Gleichheit praktisch mit Freiheit identisch ist, so deswegen, weil das Gegenteil materiell unmöglich ist, anders gesagt: Zwangsläufig werden stets beide gemeinsam angefochten. Diese These selbst ist umfangslogisch zu interpretieren: Gleichheit und Freiheit werden genau unter denselben Bedingungen, in denselben "Situationen« angefochten, weil es kein Beispiel für Bedingungen gibt, unter denen die Freiheit unterdrückt oder gehemmt und nicht auch die Gleichheit unterdrückt oder eingeschränkt, das heißt beseitigt wird, und umgekehrt. Weder von der Geschichte der kapitalistischen Ausbeutung, die in der Praxis die durch den Arbeitsvertrag proklamierte Freiheit leugnet, um bei der praktischen Negation der Freiheit zur Geltendmachung von Ansprüchen zu enden, noch von der Geschichte der sozialistischen Regime, die die öffentlichen Freiheiten unterdrücken, um bei der Errichtung einer Gesellschaft noch stärkerer Privilegien und Ungleichheiten zu enden, habe ich eine Widerlegung zu befürchten. ${ }^{19}$ offensichtlich hat die Unterscheidung von »individuellen « und »kollektiven« Freiheiten wie auch die von "formaler« und »wirklicher« Gleichheit hier keinen Sinn: Eher geht es um die Frage des Grads an Gleichheit, der für die kollektive Umsetzung individueller Freiheiten unabdingbar, und um die des Grads an Freiheit, der für die kollektive Gleichheit unerlässlich ist. Die Antwort ist jedesmal dieselbe: das Höchstmaß unter den gegebenen Voraussetzungen. Daher lässt sich der Beweis für die Proposition Égaliberté - die negative Erfahrung, der einzigmögliche, aber als solcher auch hinreichende Beweis noch auf andere Weise führen: Die unterschiedlichen Formen von sozialer »Macht» oder "Gewalt«, die den Menschen-und-Staatsbürger sei es Ungleichheiten, sei es Freiheitsbeschränkungen aussetzen, konvergieren zwangsläufig. Es gibt kein Beispiel für Restriktionen oder Entzug von Freiheit ohne soziale Ungleichheiten, und kein Beispiel für Ungleichheiten ohne Einschränkung oder Abschaffung von Freiheiten (sei es auch nur um den Widerstand in Schach zu halten), selbst wenn es Abstufungen, sekundäre Spannungen, Phasen instabilen Gleichgewichts, Kompromisssituationen gibt, in denen Ausbeutung und Herrschaft sich nicht gleichmäßig über alle verteilen. Ebendieser Mechanismus erlaubt die Herausbildung von Klassen oder herrschenden Eliten, die Macht unvermeidlich in Übermacht, in Hegemonie verwandelt.

Wenn Freiheit nämlich nicht Gleichheit ist, bedeutet sie entweder Überlegenheit, "Herrschaft", oder Unterjochung und Abhängigkeit von irgendeiner Macht, was widersinnig ist. Darum ist Gleichheit korrelativ als allgemeine Form radikaler Negation jeder Unterjochung und jeder Herrschaft zu denken, das heißt als Befreiung der Freiheit selbst von äußeren oder inneren Mächten, die sie sich aneignen und in ihr Gegenteil verkehren. ${ }^{20}$

Nun wird verständlich, warum der Text der Déclaration, ein »Gelegenheitsprodukt « der bürgerlichen Wortführer der Revolution, nicht ihre eigene Beherrschung oder Kontrolle des Prozesses, an dem sie teilnehmen, zum Hauptinhalt hat. Es wird verständlich, warum alsbald ein Kampf einsetzt, bei dem es um die Anwendung der "Prinzipien von 1789«, das heißt praktisch um ihre unbegrenzte Ausdehnung oder ihre Begrenzung geht. 
Verständlich wird aber auch, was ich gerade vorbrachte: dass die Bedeutung der Gleichung Mensch = Citoyen nicht so sehr die Definition eines politischen Rechts ist als vielmehr die Bekräftigung eines universellen Rechts auf Politik. Zumindest der Form nach - aber diese Form ist vom Schlage derer, die zur materiellen Waffe werden können - eröffnet die Déclaration eine unbegrenzte Sphäre der Politisierung von Rechtsansprüchen, die, jeder auf seine Weise, die Forderung nach Citoyenneté oder öffentlicher, institutioneller Verankerung von Freiheit und Gleichheit erneuern: Von der revolutionären Phase an machen Lohnarbeiter, Abhängige, Frauen, Sklaven, später auch Kolonisierte sich diese unbegrenzte Öffnung zu Nutze, um rechtliche Ansprüche anzumelden.

Hier aber nun der zweite Aspekt. Wesentlichen Anteil an der Wahrheit unseres Textes hat seine »negative Universalität«, das heißt seine absolute Unbestimmtheit. Da wir hier von einer Wahrheit und einem Wahrheitseffekt in der Geschichte sprechen, müssen wir die Ebene der Aussage und die ihrer Äußerung, oder anders gesagt: Bedeutung und Referenz, mehr denn je miteinander verknüpfen. Die Unbestimmtheit macht die ganze Stärke der Aussage, aber auch die praktische Schwäche ihrer Äußerung aus. Oder vielmehr: Sie bewirkt, dass die Konsequenzen der Aussage, die ja ganz und gar von "Kräfteverhältnissen" und ihrer Entwicklung unter den jeweiligen Umständen abhängen, selber unbestimmt bleiben - von Kräfteverhältnissen, in denen es gilt, die individuellen und kollektiven Bezugspunkte für die Égaliberté mit mehr oder weniger »Vorsicht« und »Augenmaß«, aber auch »Kühnheit« und »Dreistigkeit« gegenüber den etablierten Kräften praktisch $\mathrm{zu}$ konstruieren. Zwischen den Bedingungen, die historisch jene Konstruktion von Institutionen determinieren, dieder Proposition Égaliberté entsprechen, und der hyperbolischen Universalität der Aussage wird permanent Spannung herrschen. Dennoch muss sie ständig wiederholt, und zwar gleichförmig, unverändert wiederholt werden, damit der Wahrheitseffekt eintritt, ohne den es keine revolutionäre Politik gibt. Es wird also eine ständige Spannung zwischen der universell politischen Bedeutung der "Menschenrechte» und der Tatsache herrschen, dass diese Aussage es völlig der »Praxis«, dem »Kampf«, dem »sozialen Konflikt« - insbesondere der Entwicklung des Konflikts, der schon bei ihrer Formulierung Pate stand - überlässt, eine »Politik der Menschenrechte« ins Leben zu rufen. Hier wollen wir lediglich festhalten: Diese Spannung ist so groß, dass das, was den Diskursen über die "Menschenrechte" - diesen Kompromissdiskursen, die die effektive Gleichsetzung von Mensch und Staatsbürger umgehen oder verdrängen - im allgemeinen am meisten mangelt, eben die Politik der Menschen- und Staatsbürgerrechte ist.

Ich komme nun zum letzten Punkt. Ich möchte folgende Hypothese zur Diskussion stellen: Die Égaliberté näher bestimmen oder sie um den Preis von Auseinandersetzungen umsetzen, die sich konkret gegen die historischen Negationen richten, deren theoretische Negation diese Proposition selbst darstellt, heißt ihre Wahrheit umsetzen. Eine solche Umsetzung hängt aber von zwei Faktoren ab: von einer Bestimmung der realen Widersprüche der postrevolutionären Politik, das heißt der unter den jeweiligen Bedingungen, unter denen sie sich fortsetzt oder auch wieder herstellt, gegebenen Kräfteverhältnisse und Interessenkonflikte; aber auch von einer Bestimmung der Formen, unter denen solche realen Widersprüche in dem von der revolutionären Proposition geöffneten Raum denkbar sind. Oder ganz einfach und noch grundsätzlicher: von der Möglichkeit, solche Widersprüche in diesem ideologischen Raum überhaupt »adäquat« $\mathrm{zu}$ denken, das heißt sie dort $\mathrm{zu}$ benennen und ihre 
"Lösung« als Verwirklichung der Égaliberté zu formulieren. Daraus, dass die Proposition Égaliberté universell wahr ist, geht nämlich nicht hervor, dass sie »die ganze Wahrheit« ist (ein in Wahrheit widersprüchlicher Begriff). Daher die Tatsache, dass sie durch die unmittelbare Öffnung eines Gedankenraums auch dessen Schließung bestimmt, anders gesagt: ihn unmittelbar als ideologischen Raum bestimmt.

Abschließend möchte ich mich ausschließlich mit diesem zweiten Aspekt beschäftigen, und zwar aus folgendem Grund: Die historische Erfahrung hat uns nicht nur zu begreifen gezwungen, dass unterschiedliche Widersprüche, unterschiedliche Kämpfe für Gleichheit und Freiheit innerhalb des Felds der revolutionären Politik nicht spontan miteinander vereinbar sind, sondern auch, dass sie nicht in derselben Sprache, in den Elementen ein und desselben Diskurses ausgesprochen werden können, was doch eine Mindestvoraussetzung für ihre praktische Begegnung wäre - von ihrer »Verschmelzung" in ein und derselben demokratischen oder revolutionären Bewegung ganz zu schweigen. Die Gründe dafür beruhen zumindest zu einem Teil - und dieser genügt, sich von der relativen Inadäquatheit der revolutionären Idee heute Rechenschaft abzulegen - auf der Heterogenität der »Widersprüche«, mit denen wir es hier zu tun haben, und radikaler noch auf der Tatsache, dass es sich nicht um "Widersprüche" im selben Sinn des Wortes handelt. Es wird genügen, an das $\mathrm{zu}$ erinnern, was traditionell als Klassenwiderspruch und als Widerspruch im Geschlechterverhältnis (in den Beziehungen zwischen Männern und Frauen) bezeichnet wurde. Meinerseits möchte ich aus Gründen, die ich noch deutlich zu machen hoffe, einen »Widerspruch « hinzufügen, der mir ganz ebenso grundlegend und ganz ebenso heterogen $\mathrm{zu}$ sein scheint wie die vorangegangenen, und den Formulierungen wie "Trennung von Hand- und Kopfarbeit« oder Spaltung zwischen körperlicher Tätigkeit und Geist oder Denken ideologisch einordnen helfen.

Unsere Diskussion sollte daher die Form der Konstruktion einer Konfiguration oder einer Topik annehmen, der allgemeinsten Topik der ideologischen Spannungen der modernen Politik in dem Sinne, wie die revolutionäre Proposition sie umzustrukturieren bestimmt hat. Innerhalb einer solchen Topik müssen wir versuchen, die Formulierung der Widersprüche zu situieren, um ihre Heterogenität und Distanz zu ermessen.

Meine Konstruktionshypothese lautet schematisch dargestellt folgendermaßen:

1. Die Gleichsetzung von Freiheit und Gleichheit ist für die moderne, "subjektive« Umgestaltung des Rechts unerlässlich, aber nicht in der Lage, deren institutionelle Stabilität zu garantieren. Eine Vermittlung ist erforderlich, die jedoch die antithetischen Formen von »Brüderlichkeit« (oder Gemeinschaftlichkeit) und »Eigentum« annimmt.

2. Jede dieser Vermittlungen ist ihrerseits Konfliktgegenstand und praktisch geteilt: auf Seiten der Gemeinschaftlichkeit zwischen Nation und Volk, auf Seiten des Eigentums zwischen Arbeit und Kapital. Die Verbindung dieser Gegensätze ist die allgemeinste ideologische Form des »Klassenkampfs«.

3. Jede dieser Vermittlungen wie auch die Konflikte, in denen sie sich äußern, verdrängen einen anderen Typus von »Widerspruch«: die BrüderlichkeitGemeinschaftlichkeit den Geschlechterunterschied, das Eigentum (Arbeit und / oder Kapital) die Trennung zwischen "geistigem « Wissen und »körperlicher« Tätigkeit.

Dementsprechend stehen wir vor zwei völlig heterogenen Typen von Widersprüchen, die sich nicht nur nicht vereinheitlichen lassen, sondern auch - zumindest solange, wie die diskursive Matrix politischen Handelns auf dem Konzept des Menschen-und- 
Staatsbürgers beruht, von dem wir ausgegangen sind - in gewisser Weise nicht zu vereinbarende, obschon untrennbar miteinander verbundene Diskurse auslösen.

Erlauben Sie mir, die drei angesprochenen Punkte kurz zu kommentieren.

Beginnen wir mit der Frage der Vermittlungen.

Wir haben wieder von der konstitutiven Instabilität der Gleichung Mensch = Citoyen auszugehen, die auf der Identifizierung von Gleichheit und Freiheit beruht, das heißt auf der Behauptung eines potentiell universellen Rechts auf Politik. Andernorts habe ich nach anderen Autoren (und wenn man die Texte nur recht liest: nach den Revolutionären selbst) $\mathrm{zu}$ zeigen versucht, dass diese Behauptung ein unbestimmtes Schwanken, eine strukturelle Äquivokation zwischen zwei offensichtlich antinomischen »Politiken« mit sich bringt: einer Politik der Insurrektion, einer Politik der Konstitution. $^{21}$ Oder wenn man lieber will, eine Politik der permanenten, ununterbrochenen Revolution und eine Politik des Staats als institutioneller Ordnung. ${ }^{22}$ Es ist klar, dass eine solche Antinomie den Begriff der Politik selbst zerteilt, ohne dass jemals eine Synthese $\mathrm{zu}$ finden wäre (was vielleicht den typischen Charakter der Moderne ausmacht). Sie bedeutet auch, dass »Freiheit« und »Gleichheit« ständig dazu tendieren, sich $\mathrm{zu}$ entzweien, als unterschiedliche Prinzipien oder Werte in Erscheinung zu treten, auf die sich entgegengesetzte Lager oder Kräfte berufen können, sofern ihre Identität - und namentlich ihre rechtliche Identität - nicht garantiert oder, wenn man so will, auf die Einführung und den Primat eines dritten Glieds gegründet ist. Dann hätte man nicht mehr die unmittelbare Identität $G=F$, sondern eine vermittelte Identität: $G$ = F als Äußerung oder Spezifizierung eines anderen Prinzips, das zugleich als gemeinsame Essenz der beiden Elemente der Gleichung erschiene. Und gleichzeitig hätten wir die ideologische Möglichkeit, von einem negativen, unbestimmten Beweis zu einem positiven, bestimmten (aber vermittelten und infolgedessen nur eine relative Wahrheit erzeugenden) Beweis überzugehen.

Eine derartige Vermittlung kann sich indessen - gewiss aufgrund der jeder institutionellen Vermittlung sozialer, transindividueller Beziehung innewohnenden Zweideutigkeit, aber auch aufgrund der Tatsache, dass jede Institutionalisierung eines revolutionären Einschnitts dem Gesetz der »Wiederkehr des (vorrevolutionären) Verdrängten« unterliegt - nicht in einer einheitlichen Form vollziehen. Historisch nimmt sie zwei antithetische Formen an: die Vermittlung durch das Eigentum und die durch die Gemeinschaftlichkeit (die sich in der Französischen Revolution in der triadischen Formel »Freiheit - Gleichheit - Brüderlichkeit« äußert, die den drei Polen eines symbolischen Dreiecks entsprechen: Aber das Dreieck Freiheit - Gleichheit Eigentum ist nicht weniger entscheidend).

Wir können diese Konfiguration so darstellen: 


\section{$B(G)$}

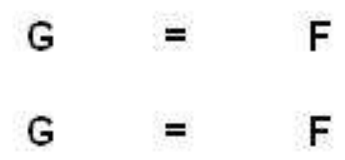

E

Halten wir hier einen Augenblick inne. Natürlich ist keiner dieser Begriffe - Freiheit, Gleichheit, Eigentum, Gemeinschaftlichkeit oder Brüderlichkeit - radikal neu. Was aber neu ist, ist ihre Gruppierung, ihre gegenseitige Definition und die Spannung, die zwischen zwei möglichen »Begründungen « für Freiheit und Gleichheit eingetreten ist, zwischen zwei alternativen Weisen, den Citoyen zu vergesellschaften: das individuelle oder kollektive Eigentum, die natürliche oder historische (oder selbst geistige) Gemeinschaft. Wir haben hier die Matrix der für die moderne Zeit charakteristischen politischen Ideologien, vom Sozialismus oder Liberalismus (die jeweils auf ihre Art das Eigentum betonen) bis zum Nationalismus und Kommunismus (die jeweils auf ihre Art die Gemeinschaftlichkeit und - namentlich in Frankreich - die Brüderlichkeit betonen). Die Prägnanz dieser Struktur feststellen heißt auch erklären, was es mit der zeitgenössischen Verlegenheit gegenüber der Politik auf sich hat. Man glaubt, sie beziehe sich auf die Begriffe Freiheit und Gleichheit: Das ist nicht sicher, eher bezieht sie sich auf deren »Komplemente«. Denn als Ort der Verankerung von Individualität, also der Beziehung Menschen / Sachen oder Mensch / Natur hat das »Eigentum« in all seinen Formen heute viel von seiner Evidenz, seiner Eindeutigkeit verloren, ist es ein komplexer, opaker Begriff geworden (was heißt es eigentlich, beispielsweise eine Fähigkeit oder eine Schuldverschreibung zu »besitzen«?). Die Brüderlichkeit/ Gemeinschaftlichkeit hat indessen sowohl ihre Eindeutigkeit verloren (denn es gibt nicht nur eine kollektivstiftende soziale Beziehung, sondern mehrere konkurrierende Gruppen oder Zugehörigkeiten, mit denen sich zu identifizieren die Individuen aufgefordert werden) als auch ihre Konsistenz (es gibt soziale Beziehungen, die die Individuen erst allzu gut gebunden haben und jetzt gar nicht mehr zu binden scheinen: zum Beispiel der Beruf, die Familie; für die Klasse und die Nation stellt die Frage sich gewiss auch immer mehr). Wir werden aber sogleich sehen, dass der tiefste Grund für dieses Wanken der »Grundlagen« der modernen Politik wohl doch in der Heraufkunft einer Politik der »Differenzen« zu suchen ist, um die es der Freiheit und der Gleichheit 
vorrangig geht und die die Identität des Eigentümers wie die des Gemeinschaftsmitglieds auflösen.

Überraschenderweise - und das ist nicht nur eine formale Symmetrie - können weder Eigentum noch Gemeinschaft ohne ein antithetisches Räsonnement Freiheit und Gleichheit »begründen« (und folglich Politiken, die auf diesen »Rechten« des Menschen-und-Staatsbürgers aufbauen). Ich möchte es das Argument der Gefahr des entgegengesetzten Übermaßes nennen. Es besagt: Das Übermaß an Gemeinschaft, der absolute Primat des Ganzen oder der Gruppe gegenüber den Individuen würde die Individualität erdrücken, und daher müssen die Beziehungen der Freiheit und Gleichheit durch das Prinzip der Garantie des Eigentums beherrscht, "gemäßigt « werden, insbesondere des Eigentums an sich selbst, an den eigenen Existenzbedingungen. Und symmetrisch dazu: Das Übermaß an Eigentum, der absolute Primat der Individualität, würde die Gemeinschaft erdrücken, und daher müssen Freiheit und Gleichheit wesentlich als Ausdrucksformen des gemeinschaftlichen Daseins der Menschen und der Institutionen definiert werden, in denen die Gemeinschaft sich verwirklicht. Dann werden die Systeme komplexer, und die alte Dialektik von Sein und Haben tritt hervor: Die Gemeinschaft beginnt dann, sich mittels einer gewissen Reglementierung des Eigentums $\mathrm{zu}$ verwirklichen, und das Eigentum mittels einer gewissen Form von Gemeinschaftlichkeit, geregelt durch Effizienz oder Justiz oder gemeinsames Interesse usw.

Vor allem aber entwickelt diese Dialektik sich nicht, ohne dass jede der beiden großen »Vermittlungen« tendenziell in sich gespalten, zweigeteilt wäre. Das liegt wohl daran, dass der Begriff universeller Citoyenneté zum Streitobjekt zwischen Herrschenden und Beherrschten und auch zwischen gewaltförmigen und rechtlichen oder legalen Formen von Politik wird - er war ja schon ursprünglich von der Konvergenz völlig heterogener sozialer Gruppen und Praktiken getragen. Auf Seiten der Herrschenden wie auf Seiten der Beherrschten wird Gewalt gegen das Gesetz, gegen die juristische Form, aber auch Legalität gegen Gewalt angedroht - ohne dass dabei übrigens eine allgemeine Regel, eine ein für allemal etablierte Anordnung der Kräfte und Ideen erkennbar wäre.

Was spielt sich historisch auf Seiten des »oberen« Dreiecks (Freiheit - Gleichheit Brüderlichkeit) ab? Schon sehr früh plagen den Konvent gleichzeitig Probleme des Kriegs im Ausland, des öffentlichen Wohls, aber auch des patriotischen Revolutionskults und der Klassenunterschiede, die, wie manche sagen, eine »neue Aristokratie«, "neue Privilegien« hervorbringen. Das System Brüderlichkeit nimmt tendenziell doppelte Gestalt an: die einer nationalen, bald auch staatlichen Brüderlichkeit und die einer revolutionären, bei der extremer Egalitarismus in Kommunismus übergeht. Das Wort Nation ändert seine Bedeutung und meint statt der Gesamtheit der Citoyens nun eine historische, auf den Staat zentrierte Zugehörigkeit. Über die Mythifizierung von Sprache, Kultur und nationalen Traditionen geht dies in seiner Extremform in die französische Variante des Nationalismus über: die Idee einer auf institutionellen Traditionen gegründeten moralischen und kulturellen Gemeinschaft. Auf der Gegenseite führt umgekehrt der Begriff »Volk« zu der allgemeinen Vorstellung vom »Proletariat« als dem Volk des Volks, dem Träger seiner Echtheit und seines wahren Gemeinschaftsstrebens. Bei Michelet und vor allem Hugo tritt diese Ambivalenz deutlich hervor.

Was geht symmetrisch auf der Gegenseite, der des »unteren« Dreiecks (Freiheit Gleichheit - Eigentum) vor sich? Auch hier ist eine Spaltung am Werk; dabei geht es um 
Fragen wie Recht auf Leben, Recht auf Arbeit. Man könnte sagen, dass es tendenziell zwei Rechtfertigungen für die Rechte des Individuums in Bezug auf das Eigentum gibt, zwei Möglichkeiten, das Individuum als Träger der Werte von Freiheit-und-Gleichheit zu denken: entweder das Eigentum an Arbeit (und namentlich die Aneignung "seiner selbst«, seiner Existenzmittel, durch Arbeit), oder das Eigentum an Kapital (ob Geldkapital oder symbolisches Kapital, beispielweise die Fähigkeit zu unternehmen, das Know-how usw.). Auf ideologischer Ebene sind diese Begriffe erstaunlich ambivalent (wie weiter oben das »Volk«). Wie der Kapitalist sich als Arbeiter, als »Unternehmer« definiert, so der Arbeiter als Träger einer Fähigkeit, eines »Humankapitals«. In beiden Fällen kann der Begriff Eigentum formal beibehalten werden, ist er doch den Ideologien des individualistischen Liberalismus und des kollektivistischen Sozialismus gleich wichtig: Beide stimmen darin überein, dass das gesellschaftlich Entscheidende das Eigentum sei.

Sehr früh schon sind diese beiden offenkundigen Widersprüche gewissermaßen politisch verschmolzen. Von 1789 bis 1793 war das Beherrschende die Frage der Gemeinschaft der Citoyens, das Problem der Brüderlichkeit, im vollständigen Wortlaut der jakobinischen Formel, die - auf akzeptable Proportionen reduziert - zur »republikanischen Devise« wurde: »Einheit Unteilbarkeit der Republik Freiheit Gleichheit Brüderlichkeit oder Tod«. Der zweite Widerspruch, der sich von 1789 bis 1795 und im Code Civil entfaltete, lief auf die symbolische Spaltung zwischen bürgerlichem Eigentum und egalitaristischem Kommunismus hinaus. Das ganze 19. Jahrhundert hindurch entwickelte sich, was ich die allgemeine ideologische Formel des Klassenkampfs genannt habe: nicht einfach nur der Gegensatz zwischen individuellem und kollektivem Eigentum, zwischen Arbeit und Kapital, sondern die Addition zweier Widersprüche. Das »bürgerliche Lager« ist, ideologisch gesehen und die materiellen Interessen einmal beiseite gelassen, eine Eigentumsform, die sich einer anderen entgegenstellt, und gleichzeitig eine Gemeinschaftsform, die sich einer anderen entgegenstellt ${ }^{23}$ : Liberalismus plus Nationalismus. Und ebenso ist das "proletarische Lager« eine Eigentumsform, die des kollektiven oder sozialen oder auch geplanten Eigentums, plus eine Gemeinschaftsform: eben der Kommunismus, Erbe des Ideals der Brüderlichkeit der revolutionären Massen und der Idee, dass Citoyens im eigentlichen Sinn die Menschen aus dem Volk sind, die Arbeiter. ${ }^{24}$

Kann man dabei stehen bleiben? Ich glaube nicht. Und dies ist einer der Gründe dessen, was ich die relative Inadäquatheit der Idee der Revolution am Ende des 20. Jahrhunderts genannt habe, die damit auf ihre eigenen Ursprünge zurückverweist. Die Widersprüche, von denen wir gesprochen haben, sind manifest, sie werden seit zwei Jahrhunderten in Diskursen erläutert, aus denen die moderne, die postrevolutionäre Politik besteht. Das heißt: Sie lassen sich vollkommen in einer Sprache der Freiheit und Gleichheit oder, wenn man lieber will, des Kampfes gegen Unterdrückung und Ungerechtigkeit formulieren. Aber heute stellen wir immer deutlicher fest, dass es noch eine andere Art von »Widersprüchen« oder "Teilungen« gibt, die sich in dieser Sprache schwer formulieren lassen (oder die in Formulierungen wie Unterdrückung und Ungerechtigkeit nie ganz aufgehen). Jedenfalls werden wir uns dessen immer stärker bewusst. Ein Zeichen der Zeit? Vielleicht.

Ich glaube, es gibt grundsätzlich zwei davon - wer in dem, was bis vor kurzem noch die »revolutionäre Partei« hieß, Politik gemacht hat, hat die eine wie die andere als nahezu unüberwindliches Hindernis bei der Herstellung einer freien Gemeinschaft von 
Individuen erfahren, die gemeinsam gegen soziale Ungleichheiten kämpfen. Denn gerade diese generell aus dem Bewusstsein und den politischen Diskursen verdrängten oder nur verschämt erwähnten Widersprüche und Teilungen einer anderen Art stellen den Begriff Individuum selbst, das Modell von Individualität oder, wenn man so will, von "menschlicher Natur« in Frage, das heißt die Möglichkeit, sich das Individuum im allgemeinen als Exemplar der menschlichen Gattung vorzustellen. ${ }^{25}$ Es handelt sich um die Geschlechterteilung (nicht nur als Teilung sozialer Rollen, sondern tiefergreifend als absolute Differenz, als Dualität von Mann und Frau, als Schnitt, der die menschliche Gattung - und damit jede Gemeinschaft - in zwei asymmetrische Hälften ohne vermittelnde Instanz zertrennt); und um die Trennung von Körper und Geist (diese "platonische« Entgegensetzung der beiden Seiten der Individualität, die doch ein Spinoza als miteinander »identisch « zu denken versucht hatte, findet sich als Teilung von »Handarbeit« und »Kopfarbeit«, Technik und Reflexion, ausführender Tätigkeit und Wissen, Sport und Kunst oder Kultur usw. im gesamten sozialen Feld wieder).

Gewiss handelt es sich um Ungleichheiten, genauer gesagt, um die zur Einrichtung von Ungleichheit - und damit auch zur Einschränkung oder Abschaffung der Freiheit einer ganzen »Klasse« der Menschheit - immer wieder beschworenen Grundlagen. Und doch steht hinter diesen Ungleichheiten ein Typus von Unterschied, der durch die Instituierung der Gleichheit nicht abzuschaffen ist. Was nicht heißt, dass nicht auch hier Gleichheit die formale Voraussetzung der Freiheit ist, sondern dass sie rein äußerlich bleibt. Und dieser Unterschied selbst ist in beiden Fällen außerordentlich zweideutig, da er einen biologischen und einen historischen Aspekt, eine doppelte Verbindung der Individualität mit dem Körper und mit der Sprache, einen realen und einen imaginären Aspekt miteinander kombiniert: Es handelt sich um die Unterschiede schlechthin, die die Wirklichkeit des Imaginären in der menschlichen Erfahrung denotieren und deswegen auch das Problem der internen Grenzen der Politik oder einer Transformation der Politik aufwerfen, die nicht nur den Menschen-und-Staatsbürger, sondern den Menschen als Subjekt von Phantasien und Wünschen einschließen würde.

Dafür scheint es im Rahmen der bloßen Égaliberté - obwohl sie eine notwendige Bedingung für die Anerkennung von Rechten ist - keine "politische Lösung" zu geben: durch »Trennung" der Gruppen ebenso wenig wie durch ihre »Fusion« (der Mythos vom totalen, manuell-intellektuellen Menschen trägt auch nicht weiter als der vom androgynen, mit dem er übrigens in Verbindung steht). Die moderne Politik wird von verdrängten Widersprüchen durchzogen, die, obgleich immerzu als äußerlich hingestellt, ihre diskursive, legislatorische, organisatorische, repressive Praxis indirekt prägen. Vielleicht lässt sich der Beginn ihrer eigenen Formulierung erst von heute her datieren, in dem Maß, wie die Inadäquatheit spezialisierter Diskurse über Familie, Erziehung, Berufsausbildung manifest wird.

Vielleicht können wir auch heute erst die Frage nach einer Mutation der Politik aufwerfen, die eine politische Anerkennung des Geschlechterunterschieds und des kulturellen oder geistigen Unterschieds - in ihrer Unterschiedlichkeit selbst implizieren würde, das heißt eine tatsächliche Ausdehnung des »Rechts auf Politik« auf Frauen und "Handarbeiter" und andere "Ungebildete" oder "Unwissende«, die der faktischen Notwendigkeit ein Ende bereiten würde, sich innerhalb der Politik von anderen vertreten oder lenken zu lassen. Und doch sind diese Unterschiede von Anbeginn an konstitutiv für die politische Institution als Sphäre der »Regierung«, und sie sind seit der Französischen Revolution explizit in Frage gestellt worden: sei es in 
Form der Forderung nach aktiver Staatsbürgerschaft für Frauen, sei es unter den scheinbar einander widersprechenden Formen der Forderung nach dem Recht auf Bildung und zugleich des Protests gegen die Macht der Intellektuellen, der "Wortführer« und Techniker. Die beiden Jahrhunderte seit 1789 erscheinen damit sowohl als Epoche der Verdrängung des Unterschieds (in den "Haushalt«, in die "Schule«) als auch seines unaufhaltsamen Manifestwerdens.

Obgleich diese Unterschiede allemal innerhalb einer Machtbeziehung auftreten, haben sie negativ miteinander gemein, anderes als Ungleichheit darzustellen. Genauer gesagt: Sie wohnen einer Beziehung kollektiver Ungleichheit (Männer und Frauen, Eliten und Massen) inne, die als persönliche, besondere Beziehung von Individuum zu Individuum reproduziert, ausgeübt und verifiziert wird, während die moderne Gesellschaft jede Abhängigkeit des Menschen vom Menschen formal abgeschafft hat. Deswegen erscheinen sie gegenüber dem Begriff rechtlicher und statusmäßiger Ungleichheit stets gleichsam versetzt: diesseits oder jenseits des "Sozialen«, befangen in der Kontingenz individueller Prägungen oder der Zwangsläufigkeit transindividueller Schicksale. Positiv haben sie miteinander gemein, dass sie ihre Befreiung als $»$ Recht auf Differenz in der Gleichheit« suchen müssen, das heißt nicht in der Wiederherstellung einer ursprünglichen Identität oder Neutralisierung der Differenzen in rechtlicher Gleichheit, sondern in der Herstellung einer noch nie dagewesenen, unter kein Modell subsumierbaren Gleichheit, die die Differenz selbst, die Komplementarität und Reziprozität der Besonderheiten darstellt. In gewissem Sinn ist eine solche Reziprozität in der Proposition Égaliberté schon virtuell enthalten, sie kann aber - paradoxerweise sich nur unter der Bedingung darauf berufen, dass sie die Frage der Identität von "Mensch" und "Citoyen « neu stellt: nicht um auf die Idee einer anthropologischen Differenzen untergeordneten Staatsbürgerschaft zurückzufallen (wie in deren antiker Fassung), sondern um zu einer durch anthropologische Differenz überdeterminierten Staatsbürgerschaft fortzuschreiten, hin zu einer Transformation dieser Differenz, die weder auf ihre institutionelle Naturalisierung noch auf eine Leugnung oder formale Neutralisierung hinausliefe (die faktisch doch als permanentes Mittel zu ihrer Naturalisierung funktioniert).

Trotzdem gleichen diese beiden »Differenzen« einander nicht. Die Gewalt, die von ihnen ausgeht (und die als einseitiger Ausdruck einer "Kraft«, einer transindividuellen »Macht" erscheint), unterwirft nicht dieselben Individuen, oder vielmehr nicht dieselben "Klassen« von Individuen, und vor allem unterwirft sie sie nicht mit denselben Mitteln, obwohl diese sich stets addiert haben. ${ }^{26}$

So lässt sich zeigen, dass eine Form der Monopolisierung des Wissens (oder seiner Vermännlichung oder der Instituierung spezifisch "männlichen « Wissens) stets in dem Maße an der Ungleichheit der Geschlechter beteiligt ist, wie diese nicht nur das "private«, "häusliche« Verhalten, sondern auch das »öffentliche« betrifft (oder auf einem politischen Zwang beruht, der sich im Privaten auswirkt), und dies um so grundlegender, als sie der alltäglichen Gewalt eines die Körper dominierenden Worts innewohnt. Ebenso lässt sich zeigen - wenn auch schwieriger: sei es, weil das Motiv dafür weiter verdrängt ist, sei es, weil die Sprache der Unwissenden es in unseren zeitgenössischen "technologischen" Gesellschaften noch schwerer hat als die der Frauen, sich Gehör zu verschaffen -, dass das politische Monopol der Bildung, des Sachverstands, der Meritokratie stets unter sehr ambivalenten Formen ein Modell männlicher Herrschaft impliziert: Verdrängung "weiblicher Probleme« aus dem Feld 
der anerkannten intellektuellen Disziplinen, vor allem aber Kompensierung der "geistigen « Macht, die manche Männer über andere ausüben, durch die »körperliche«, die geistig dominierte Männer über »ihre« Frauen ausüben. Dies alles hindert jedoch nicht, dass die Differenz, die Triebfeder all dieser Machtverhältnisse, auf kein Einheitsmodell reduzierbar ist.

Im Geschlechterunterschied haben wir es mit einem Zusatz an Besonderheit zu tun, der es verbietet, der Freiheit der Männer und der Freiheit der Frauen denselben Inhalt zuzusprechen und sie also auf ein Modell gemeinsamer Subjektivität zu reduzieren. Man kann als Voraussetzung ihrer Handlungsfreiheit wollen, dass Frauen über "gleiche Rechte«, gleichen Zugang zum Wissen, zur Berufsausübung, zu öffentlichen Ämtern verfügen (was eine mehr oder weniger tiefgreifende Transformation der Bedingungen ihrer Ausübung voraussetzt); man darf nicht meinen, dass sie von da an als Individuen schlechthin agieren. Gleichheit ist hier nicht Neutralisierung der Differenzen (Egalisierung), sondern Bedingung und Postulat der Diversifizierung der Freiheiten.

Bei der Ungleichheit an Wissen hingegen, die zugleich Reproduktion der Differenz zwischen einer "Masse« und einer "Elite«, Einsatz der Bildungsinstitutionen zur Einzäunung und Hierarchisierung sozialer Aktivitäten, Legitimation "geistiger« Lebensart (sei sie auch rein formal, jedem Erwerb wirklicher Kenntnisse äußerlich) als Voraussetzung und Berufung zu Autorität über »manueller « Lebensart ist, haben wir es eher mit einem Abzug von Besonderheit zu tun. Geht man (hier mit Spinoza) davon aus, dass Individualität eine Funktion der Kommunikation ist und Kommunikation sich optimal nicht zwischen Typen oder vorbestimmten sozialen Rollen entwickelt, sondern zwischen Besonderheiten, "praktischen " Erfahrungen, von denen jede von den anderen lernen und die anderen belehren kann, dann ist zuzugeben, dass die Erweiterung von Wissen als Herrschaftsträger paradoxerweise entindividualisiert. Die Universalität der Wissensfunktion in den modernen Gesellschaften, eine positive Bedingung der Konstituierung gemeinsamer politischer Sprache (und auch ihrer »Laizisierung"), wird mit einer Einschränkung realer Kommunikationsmöglichkeiten bezahlt - deren institutionelle Form eben das Monopol auf "Kommunikationsaufgaben" ist. Unter diesem Gesichtspunkt schafft die Ungleichheit eine praktisch unreduzierbare Differenz, aber der Kampf gegen Ungleichheit kann weder zur Beseitigung der Differenzen führen noch dazu, sie in Form einer verallgemeinerten Selektion der Individuen auf »demokratische« Weise zu reproduzieren. Wenn eine größere Freiheit der Individuen wie auch der Kommunikation selbst sich entwickeln soll, muss eine Neutralisierung und gleichzeitig Umverteilung des Wissens, eine "Äquivalenz« zwischen gebildeten und unwissenden Individuen im Hinblick auf das Recht zur Äußerung in der Öffentlichkeit und eine symbolische Dissoziierung der institutionellen Äquivalenz zwischen »Intelligenz« und »Wissen« erfolgen. Diese Gleichheitsforderung blieb in Wahrheit stets die Aporie der politischen Utopien, denen es um geistige Emanzipation ging. ${ }^{27}$

Diese Dissymetrie von sexueller und intellektueller Differenz schreibt sich als »konkrete Politik« der Beziehung zwischen den entscheidenden Institutionen der modernen Politik ein, der Familie und der Schule. Sie macht verständlich, warum die Familie (eine konstitutiv inegalitäre Institution) bei der Neutralisierung der intellektuellen Differenzen als Machtfaktoren zwischen "Eltern« eine schwache, "private« Rolle spielt, während die (formal egalitäre Institution) Schule eine vergleichsweise sehr große, öffentliche Rolle bei der Befreiung der Frauen spielt, 
freilich auf Kosten einer anhaltenden Tendenz zur Neutralisierung geschlechtlicher Subjektivität. Das Gegenstück zu dieser Rolle ist offenbar eine Schwierigkeit, den auf "unwissenden Frauen « ungleich stärker als auf "gebildeten Frauen« lastenden Einfluss männlicher Macht zu lockern - und dies trotz der Möglichkeiten, die Arbeit, gewerkschaftliche Tätigkeit, ja selbst die organisierte Politik bieten. ${ }^{28}$

Aus diesen Betrachtungen leite ich im Hinblick auf die Einschreibung der "anthropologischen Differenzen" in die Topik der Égaliberté folgende Vermutung ab: Man kann wohl vertreten, dass die sexuelle ebenso wie die intellektuelle Differenz Funktionen der "Gemeinschaft" sind, deren konflikthafter Charakter das Auseinanderbrechen ihrer Einheit virtuell zum Prinzip hat und nahezu ihre "Unmöglichkeit« dartut. Und dieser Widerspruch reproduziert sich kreisförmig: Wenn die Kommunikation zwischen Männern und Frauen, Intellektuellen und Handarbeitern in gewisser Hinsicht unmöglich ist, muss sie dann durch ein zusätzliches Maß an Gemeinschaftsorganisation, durch soziale Rollenteilung, durch Ämter und Würden, die zwangsläufig die Form von Ungleichheit oder Macht annehmen, erzwungen werden? Oder stellt sich die Kommunikation zwischen unterschiedlichen menschlichen Besonderheiten gerade deswegen als nahezu unmöglich heraus, weil die organische, Identität verleihende Gemeinschaft (die "Gesellschaft", der "Staat", die "Nation«, die "Klasse«) die Gleichheit in so enge Grenzen fasst, dass die Ungleichheit der Geschlechter und der ungleiche Zugang zur intellektuellen Macht weiterbestehen und sogar freien Lauf erhalten können? Aber auch hier steckt hinter der Analogie eine Dissymetrie. Mir scheint, die sexuelle Differenz unterhält eine privilegierte Beziehung zur Institution der Gemeinschaft, während die intellektuelle Differenz ihre ganze kritische Bedeutung für die Politik aus ihrer Beziehung zur Institution Eigentum bezieht.

Sobald alle Individuen als freie und gleichberechtigte Staatsbürger gelten und virtuell die Tatsächlichkeit dieser Rechte einfordern, wird die Teilung der Geschlechterrollen unmittelbar zur Notwendigkeit, damit die Gesellschaft sich als "Gemeinschaft" darstellen kann (und nicht als ein Nebeneinander »bindungsloser« Individuen). ${ }^{29} \mathrm{Zwar}$ lässt sich die These vertreten, dass jede historische Gemeinschaft als zugleich reale und imaginäre Institution auf der Beziehung der Geschlechter beruht (das heißt auf Verwandtschaft, Teilung der Aufgaben und Rollen zwischen Männern und Frauen, Bestimmung des symbolischen "Charakters« eines jeden bei Verdrängung von Bisexualität). Da aber die moderne politische Gemeinschaft nicht nur ein Staat ist, sondern ein Staat, dessen Rechtsform auf der Proposition Égaliberté beruht, stellt sie als solcher niemals eine geschlechtlich differenzierte Gemeinschaft dar: Als nationale Gemeinschaft ${ }^{30}$ basiert sie daher nicht auf der einfachen Beziehung der Geschlechter (sie ist keine erweiterte Familie, außer im metaphorischen Sinn), sondern auf praktischem und ideologischem Sexismus als Struktur des inneren, auf die ganze Gesellschaft ausgedehnten Ausschlusses der Frauen; also auf dem instabilen Gleichgewicht von Verleugnung und Universalisierung der Geschlechterdifferenz. Damit wird deren Geltendmachen als politische Kraft zum heikelsten Punkt der Gemeinschaftskrise (oder der Krise der gemeinschaftlichen Identität).

Auf der Gegenseite unterhält die intellektuelle Differenz eine privilegierte Beziehung zum Eigentum als sozialer Vermittlungsinstanz. Es ließe sich zeigen, dass der Begriff geistige Fähigkeit (im weiten Sinn, unter Einschluss des Wissens und der Willenskraft) als (ontologische) Differenz zwischen menschlicher Persönlichkeit und einem »Körper«, der 
an sich nur "Ding" ist, immer in der Vorstellung von einer geistigen Aneignung der Dinge inbegriffen war. Das zeigt negativ auch die Gesetzgebung, die Unmündigkeit oder geistige Verwirrung konstant mit der Unfähigkeit zu besitzen identifiziert. Um Dinge zu besitzen, muss man sich nämlich zunächst einmal »selbst besitzen«, und dieser Besitz ist nichts anderes als der Oberbegriff für Intelligenz. Wenn indes das individuelle oder kollektive - Eigentum gegenüber der Gemeinschaft zur Vermittlungsinstanz von Gleichheit und Freiheit, zur Voraussetzung individueller Menschenwürde und Bedingung von Citoyenneté wird, ändert diese Fähigkeit / Unfähigkeit ihre Bedeutung: Noch einmal tritt sie aus der rein "privaten« Sphäre heraus, um "öffentlichen" Wert zu erwerben. Alles Eigentum untersteht den durch das Wissen der politischen Ökonomie formalisierten Codes und Äquivalenzen; jedes Individuum ist »Eigentümer« (und wird an seinem Eigentum gemessen), insofern es die theoretische und praktische Wissenschaft des Wertetauschs kennt oder selbst von ihr gekannt und anerkannt ist (das heißt selbst in ihrer Buchhaltung vorkommt). Nur vermittels dieses abstrakten Wissens, das sich immer mehr autonomisiert und »intellektualisiert«, während es sich zugleich immer mehr »materialisiert«, stehen ein Individuum oder eine Klasse noch in Beziehung zu ihrem eigenen Sein und Haben. Dieser Prozess der Autonomisierung-Intellektualisierung-Materialisierung des "Wissens" determiniert immer direkter die Ausübung des »Eigentumsrechts« und folglich die Individualität. Aber gleichzeitig macht er die Identität der Eigentümer, die Identität des "Subjekts« des Eigentums immer ungewisser.

Dieser Prozess überschneidet sich mit dem Klassenkampf. Bekanntlich wird mit der Konzentration des Kapitals die (reale oder angenommene) technische »Kompetenz« auf Kosten von auf Erbschaft oder »persönlicher« Tätigkeit gegründeten Ansprüchen zur Bedingung seiner Aneignung. Fraglich ist auch, ob diese Entwicklung nicht einer vollständigen Verkehrung des Rechts der Menschen auf Eigentum in eine Vereinnahmung der Menschen (und Citoyens) durch das sich bewegende Wertsymbol (das Geld oder die Akkumulation) entspricht: eine Beschreibung, die für den "Kapitalisten« noch mehr zuträfe als für den »Lohnempfänger«. Korrelativ dazu hat die Ausbeutung der Lohnarbeit bekanntlich immer mehr die Entpersönlichung des Arbeiters in einer entintellektualisierten Masse zum Resultat ${ }^{31}$, und die Akkumulation der "geistigen Potenzen" der Produktion und des Austauschs außerhalb der ausführenden Tätigkeit der Individuen (heute in der sich selbst reproduzierenden Gesamtheit der Computerhard- und -software) zur Bedingung. Eben diese Spaltung zwischen geistiger und körperlicher Arbeit identifizierte Marx in der tiefsten Schicht seiner Analyse des Eigentumsverhältnisses als Ausbeutungsverhältnis als den Punkt, an dem Ausbeutung und Herrschaft (oder "Entfremdung «) miteinander verschmelzen. Für den Arbeiter, dessen Aufgabe (und folglich auch Ausbildung) völlig entintellektualisiert ist, ist der "Besitz seiner selbst« oder seiner "Arbeitskraft« zur totalen Fiktion geworden. Aber man muss wohl einen Schritt weiter gehen und die Autonomisierung des Wissens über die individuelle Intelligenz hinaus (und also auch über die Gestalt des Intellektuellen hinaus) als Auftakt zu einer potentiellen Krise jeder Möglichkeit für das Individuum oder das Kollektiv bezeichnen, sich als "Eigentümer« irgendeines Dings und seiner selbst vorzustellen. Dann haben wir es nicht allein mit einem Mechanismus zur Trennung der menschlichen Natur zu tun, der der Forderung nach Freiheit und Gleichheit praktisch widerspricht (und den man mehr oder weniger leicht diesseits der Politik, im "Ökonomischen", "Sozialen« und "Kulturellen« ansiedeln und dazu ausnutzen könnte, die Monopole politischer Vertretung zu perpetuieren); wir haben es 
mit einer Auflösung der politischen Individualität zu tun: Das »Recht des Citoyen« findet sich, soweit es von Eigentümern ausgeübt werden soll, seiner Substanz entleert, während die Frage der Gleichheit und Freiheit auf ihre ursprüngliche Formulierung zurückgeworfen ist, ohne dass eine Antwort dafür absehbar wäre: Was für »Menschen« sind eigentlich die Citoyens?

Somit ließe sich aus der anfänglichen Topik eine andere ableiten, die sich allmählich Bahn bricht, gewissermaßen ihre Kehrseite oder die Wiederkehr des Verdrängten:

\author{
Männer /I Frauen \\ (männlich /I weiblich)
}

\author{
$G=F$ \\ (geistig /I körperlich) \\ Gebildete II Unwissende
}

Statt "Vermittlungen" für die Instituierung von Égaliberté und ihre ideologische Begründung benennt diese Topik Punkte der Ungewissheit für die vorangegangenen Vermittlungen und Begründungen, Punkte, die mit der anthropologischen Differenz gleichzeitig die moderne individuelle und gemeinschaftliche Identität ins Wanken bringen. Diese Punkte erheben maximalen Anspruch auf Gleichheit und Freiheit (oder gleiche Freiheit), aber die konkreten (ob rechtlichen oder praktischen) Formen ihrer Befriedigung sind heute in keiner Weise abzusehen. Für eine Neugestaltung der Politik sind dies daher die heiklen Punkte schlechthin.

Indem wir diese Punkte zu der universellen Wahrheit in Bezug setzen, die der Text der Déclaration von 1789 enthält, sind wir mit der Entfaltung der historischen und ideologischen "Dialektik« der Égaliberté an ein Ende gelangt, das nicht ein Ende der Geschichte darstellt, sondern eine Frage, die in der Geschichte und durch sie im Hinblick auf ihre Fortsetzung gestellt ist. Diese Dialektik hat uns erlaubt, nacheinander zu situieren:

- die der revolutionären Politik inhärente Alternative (nicht so sehr die von Gewalt und Recht als vielmehr die von Insurrektion und Konstitution, oder, um mit Lenin $\mathrm{zu}$ sprechen, von Staat und Nichtstaat);

- den postrevolutionären Widerspruch zwischen einer "formalen« Instituierung von Freiheit und Gleichheit und ihrer »Verwirklichung« in den Formen von Eigentum und 
Gemeinschaft - einen Widerspruch, dessen allgemeinste Form der Klassenkampf ist (Sozialismus gegen Liberalismus, Kommunismus gegen Nationalismus);

- schließlich die anthropologischen Differenzen, die in das Feld der Politik zurückkehren und damit ihre gegenwärtige Ungewissheit "produzieren«: Ungewissheit bezüglich ihrer Sprache, ihrer Themen und ihrer Ziele.

Diese Dialektik erlaubt uns auch, drei »Epochen« der Politik nacheinander zu situieren: eine alte Epoche, in der der Begriff vom Staatsbürger anthropologischen Unterschieden, dem unterschiedlichen Status von freiem Menschen und Sklaven, von Herrscher und Untertan, von "mündiger" und "unmündiger" Menschheit untergeordnet ist; eine moderne Epoche, in der die Begriffe Mensch und Citoyen einander virtuell gleichgesetzt sind und die allen Menschen das Recht auf Politik eröffnet; schließlich eine postmoderne Epoche, in der die Frage nach einer Überwindung des abstrakten oder allgemeinen Begriffs vom Menschen auf der Basis verallgemeinerter Staatsbürgerschaft gestellt ist. Freilich ist zu bemerken, dass die Epochen zwar aufeinander folgen oder einander hervorbringen, aber nicht wie die Szenen eines Theaterstücks einander ablösen: Für uns und folglich für unsere Beziehung zur politischen Frage sind sie in zerfallener Totalität, in einer "Unzeitgemäßheit", die eben die Struktur des "gegenwärtigen Augenblicks« ist, noch alle gegenwärtig, was besagt: Wir haben es gleichzeitig mit dem Staat, dem Klassenkampf und der anthropologischen Differenz zu tun. Unsere Aufgabe besteht darin, ein praktisches Verhalten auf all diesen Ebenen zugleich zu konstruieren, ohne ihre Synthese herstellen zu können. Das heißt jedoch nicht, dass wir über keinen roten Faden verfügen. An der Nahtstelle zwischen »alter« und »moderner« Politik fanden wir die im revolutionären Schnitt implizierte Rechtstatsache: die Proposition Égaliberté und ihren universellen Wahrheitseffekt. An der Nahtstelle zwischen der modernen Politik und der, die aus ihr heraus und gegen sie zu entstehen im Begriff ist, finden wir die Problematik einer Neugestaltung: Wie können wir von der universellen zur besonderen Wahrheit gelangen, wie den Besonderheiten das Programm und selbst den Namen der Égaliberté einschreiben? Von jener Tatsache zu diesem Problem gibt es keine Kontinuität, kein einfaches Fortschreiten, erst recht keine Ableitung. Aber es gibt zwangsläufig einen Zusammenhang, denn ohne die Tatsache würde das Problem sich nicht einmal stellen.

\section{BIBLIOGRAPHIE}

Balibar, É. (1989): „Citoyen sujet - Réponse à la question de Jean-Luc Nancy: qui vient après le sujet?«, in: Cahiers Confrontation, 20, S. 23-47.

Balibar, É. (1992): Les frontières de la démocratie, Paris, La Découverte.

Bredin, J.D. (1988): »Enfants de la science, question de conscience«, in: Le monde, 27. 2. 1988.

de Baecque, A. / Schmale, W. / Vovelle, M. (1988): L'an 1 des Droits de l'Homme, Paris, Presses du C.N.R.S. 
Gauchet, M. (1989): La Révolution des Droits de l'Homme, Paris, Gallimard.

Gauthier, F. (1988a): »Les déclarations du droit naturel 1789-1793«, in: Vovelle, M. (Hg.): L'état de la France pendant la Révolution, Paris, La Découverte, S. 416-420.

Gauthier, F. (1988b): „De Mably à Robespierre. De la critique de l'économique à la critique du politique«, in: Thompson, E.P. et al. (Hg.): La guerre du blé au XVIIIe siècle, Paris, Éditions de la Passion, S. 111-144.

Gauthier, F. (1989): »Le droit naturel en Révolution«, in: Balibar, E. / Beck, J.-S. / Bensaïd, D. / Berger, D. et al.: Permanences de la Révolution, Paris, La Brèche.

Gauthier, F. (1992): Triomphe et Mort du Droit naturel en Révolution, Paris, PUF.

Marx, K. (1844): »Zur Judenfrage«, in: Ruge, A. / Marx, K. (Hg.): Deutsch-Französische Jahrbücher, 12, Paris.

Milner, J.-C. (1983): Les noms indistincts, Paris, Eds. du Seuil.

Rancière, J. (2007 [1987]): Der unwissende Lehrmeister. Fünf Lektionen über die intellektuelle

Emanzipation. Aus dem Französischen von Richard Steurer, Wien, Passagen Verlag.

Schwartz, O. (1990): Le monde privé des ouvriers - Hommes et Femmes du Nord, Paris, PUF.

\section{NOTES}

1. Im qualitativen Sinn des Wortes, wie Kant es benutzt, und nahe dem, was Gramsci "subalterne« Gruppen nennt.

2. Im Rückblick scheinen Marat und Saint-Just damit Benjamin Constant an »Liberalität« zu überbieten!

3. Halten wir im Vorgriff auf spätere Ausführungen die enge Verbindung zwischen der Vorstellung von den Menschenrechten als (bloßem) »Ideal« und der Idee fest, sie seien im wesentlichen - und im Rahmen des Möglichen - durch eine öffentliche Macht zu gewähren und zu gewährleisten.

4. Bredin (1988) in einer Rezension zu François Terré, L'enfant de l'esclave.

5. Vgl. meine frühere Untersuchung: Balibar (1989).

6. Gauchet (1989).

7. Gauthier (1988a, 1988b, 1989, 1992).

8. Balibar (1989).

9. Ich tue F. Gauthiers Idee - deren Stärke ich anerkenne, obwohl ich anderer Auffassung bin wohl kaum Gewalt an, wenn ich sie folgendermaßen umformuliere: Von 1789 bis 1793 haben die revolutionären Wortführer die Form der Déclaration des Naturrechts stets beibehalten, die mit der herkömmlichen Legitimation der Macht bricht; sie haben jedoch ihren Inhalt vertieft, um ihn mit dieser Form in Einklang zu bringen, und sind dabei über die Formulierung des ursprünglichen Kompromisses hinausgegangen (vor allem bezüglich des "unverletzlichen und heiligen« Charakters des Eigentums): Damit haben sie sich vom ursprünglichen Kern der Konzeption des Naturrechts der Individuen - wie es beispielsweise bei Locke zu finden ist, bei dem das einzige universelle Eigentum der »Besitz seiner selbst« ist, aus dem alle anderen Eigentumsformen hervorgehen - nicht entfernt, vielmehr sich ihm weiter angenähert. Das »Recht auf Leben« ist somit die Wahrheit des jakobinischen Egalitarismus und gleichzeitig das Kriterium der Adäquation von Form und Inhalt der Menschenrechte. Nebenbei bemerkt ist dies in etwa auch 
die Lesart, die Fichte in seinen Beiträgen zur Berichtigung der Urteile des Publikums über die französische Revolution vertritt.

10. Balibar (1989).

11. Die Ausarbeitung der "Artikel« der Déclaration führt erst am Tag nach der Nacht des 4. August zu einer Lösung [In der Nacht des 4. August 1789 hob die Assemblée constituante die feudalen Standesrechte auf. A.d.Ü.]. M. Gauchet hat Recht, wenn er die unter diesen Umständen eintretende "Neutralisierung « der ideologischen Unvereinbarkeiten und die "Anonymisierung" der von den verschiedenen revolutionären Ideologen vorgelegten persönlichen Entwürfe hervorhebt.

12. Dass die Schlussredaktion der Déclaration den Vertrag ausspart, indiziert deutlich ihre Distanz von den naturrechtlichen Ursprüngen und hängt eng zusammen mit der (provisorischen) Aufgabe der Idee einer Erklärung der Rechte und Pflichten. "Pflichten« sind nämlich das Gegenstück zu »Rechten«, wenn man Gegenseitigkeit zwischen zwei Parteien voraussetzt: entweder zwischen den Individuen und »ihnen selbst« oder zwischen Individuen und der "Gemeinschaft«, der »Gesellschaft" oder dem »Staat «.

13. Ich behaupte nicht, dass diese Tradition willkürlich sei: Nicht allein spiegelt sie den politisch-juristischen Gebrauch, den man von der Déclaration macht und über den man sich Rechenschaft ablegen muss, sie wurzelt auch in den erklärten Absichten eines großen Teils ihrer Verfasser (vgl. Gauchet [1989], und de Baecque / Vovelle / Schmale [1988]). Der Ausdruck dieser Absichten ist eng mit der Tatsache verbunden, dass die Verfasser die Formulierung der Rechte diskutieren und über sie streiten im Hinblick auf die Verfassung, der sie »eine Grundlage geben« sollen. Bezeichnenderweise zögern sie aus theoretischen Gründen ebenso wie aus Gründen politischer Opportunität unablässig, eine klare Trennungslinie zu ziehen zwischen dem, was zur Déclaration des Droits (also der Grundlage) gehört und dem, was der (auf ihr »begründeten«) Verfassung vorbehalten bleiben soll, anders gesagt: den Punkt zu fixieren, an dem Staatsbürgerrechte beginnen, die keine (universellen) Menschenrechte sind. Hier müssen wir also damit beginnen, zwischen Absicht und Verwendung des Textes einerseits und dem Text selbst einschließlich seiner virtuellen, von den Absichten unabhängigen Auswirkungen andererseits sorgfältig zu unterscheiden. Fügen wir hinzu, dass die Wahrnehmung eines grundsätzlichen oder graduellen Unterschieds zwischen "Menschenrechten « und "Staatsbürgerrechten « durch ihre Verwendung im Feld des internationalen Rechts und der zeitgenössischen internationalen Politik verstärkt wurde und die ursprüngliche Idee einer »Grundlegung« dabei fast umgekehrt worden ist: Im Wesentlichen gilt heute als "Menschenrechte«, was den Individuen außerhalb des staatlichen Rahmens zuerkannt wird, unabhängig davon, dass sie Bürger dieses oder jenes (National-)Staates sind; aber praktisch auch das, was ihnen durch demokratische (National-)Staaten zuerkannt wird (und in dieser Hinsicht beruhen sie auf der historischen Existenz einer (itoyenneté). Hier ist es auch angezeigt zu vermerken, dass die Formulierungen der Déclaration ihrem Wortlaut nach und trotz des Bezugs auf die »Nation", auf den ich zurückkommen werde (»Das Prinzip aller Souveränität beruht wesentlich auf der Nation«), weder nationalistisch noch kosmopolitisch sind, und grundsätzlicher noch: dass das Konzept »Citoyen«, dem sie Gestalt verleihen, nichts mit Zugehörigkeit zu tun hat. Es handelt sich nicht um den Begriff eines »Bürgers aus« diesem oder jenem Staat, dieser oder jener Stadt oder Gemeinschaft, sondern gewissermaßen um den Begriff eines »Staatsbürgers im absoluten Sinn«.

14. Was in dem definitiv am 26. August 1789 angenommenen (und «am 5. Oktober 1789 vom König gebilligten«) Text offensichtlich der Idee einer Grundlegung zuzurechnen ist, ist die Präambel. Sie beinhaltet sogar drei grundlegende Bezugspunkte: das revolutionäre politische Ereignis, die Natur, das höchste Wesen.

15. Bekanntlich war und blieb die Aufnahme dieses Rechts Gegenstand hitzigster Kontroversen: sowohl zwischen Parteigängern der "Ordnung« (die 1795 seine Abschaffung erreichten) und denen der »ununterbrochenen Revolution« (die 1793 versuchten, seine entscheidende Funktion 
zu unterstreichen) als auch zwischen Befürwortern der juristischen Logik (es sei »widersprüchlich« für einen Rechtsstaat, seine eigene Negation zu kodifizieren) und Fürsprechern der sozialen Logik (es sei »widersprüchlich« für kollektiv souveräne Individuen, nicht zu bestätigen, dass jede Regierung, jede Institution von ihrer Freiheit abhängen). Die Festschreibung des »Rechts auf Widerstand« in den Grundrechten bestätigt daher voll und ganz, dass die Modalität, mit der wir es hier zu tun haben, die einer Einheit von Gegensätzen ist.

16. Zum Beispiel das, nur von seinesgleichen verurteilt zu werden, Gegenstand der berühmten provocatio römischer Bürger, die der Bericht über den Heiligen Paulus unserem Kulturkreis überliefert hat: civis romanus sum!

17. Marx (1844). [A.d.Ü.]

18. Oder vielmehr im franko-lateinischen "Kolinguismus« als aequalibertas, damit in allen europäischen Sprachen verständlich, also heute in allen Weltsprachen.

19. Was zur Zeit im Osten vorgeht, dürfte zumindest einem bei der »Rechten« wie bei der "Linken« gleich beliebten Mythos ein Ende bereitet haben: dem gleicher, aber unfreier Gesellschaften (der unter der Hand zu verstehen gibt, es existierten freie Gesellschaften ohne allzu viel Gleichheit).

20. Heißt das Befreiung von jedem Gesetz? Anders gesagt, will die Égaliberté die Freiheit ohne Gesetz, ohne »Regel«, »Zwang« oder »Grenze«? Alle antiegalitäre Argumentation findet hierin ihren gemeinsamen Fundus. Und das wirft die Frage danach auf, was unter »Gesetz« zu verstehen ist: Was wäre ein Gesetz ohne bindende Autorität, ohne "Souveränität«? Erneut ein innerer Widerspruch? Von Anfang an hat diese Frage die revolutionäre Erfahrung nicht ruhen lassen.

21. Vgl. meine bereits erwähnte Studie, Balibar (1989).

22. Dies hängt auch mit dem zusammen, was wir oben feststellten (und was eine der stärksten Bedeutungen der Idee der Insurrektion ist, ein ursprüngliches Band zwischen Recht auf Insurrektion und generalisierter Citoyenneté): $\mathrm{G}=\mathrm{F}$ heißt, dass die Menschen sich selbst emanzipieren, dass niemand durch einen anderen befreit werden kann, dass das Recht auf Politik unbegrenzt und überall auszuüben ist, wo eine Autorität herrscht, die Menschen oder Menschengruppen wie Unmündige zu behandeln beansprucht.

23. Was Marx nicht recht verstanden hat, weil er die Gemeinschaft allein im revolutionären Lager sah: Das liegt daran, dass Marx zuerst Kommunist, dann erst Sozialist war.

24. In dieser Hinsicht ist das »Wortspiel« wesentlich, auf dem sich der Gegensatz von revolutionärem Kommunismus und Liberalismus begründet: Ist die Qualität »aktiver Staatsbürger« den Eigentümern, den Unternehmern, den »Fähigen« vorzubehalten oder durch die Aktivität zu definieren, die Arbeit und Initiative zur kollektiven Emanzipation ineins ist, »Assoziation«? Vergessen wir nicht die "pathologischen« Varianten dieser ideologischen Konfigurationen, mit denen Liberale wie Sozialisten und Kommunisten stets ähnlich große Verständnisprobleme hatten, zum Beispiel die Addition von Kollektivismus und Nationalismus oder von revolutionärem Messianismus und sozialem Konservatismus.

25. Auf deren Verleugnung beruht der Naturalismus konservativer »bürgerlicher« Ideologien (insbesondere der biologisierende Naturalismus des sozialen Darwinismus, des Rassismus usw.), aber auch der mehr oder weniger messianische Antinaturalismus revolutionärer antibürgerlicher Ideologien (die die christliche Thematik des »neuen Menschen« transponieren).

26. Ich setze "Klassen« in Anführungszeichen, weil es sich hier typischerweise um jene ungebundenen Kollektive handelt, die Jean Claude Milner in Les mots indistincts "paradoxe Klassen" nennt. So bilden die Frauen keine geschlossene Gruppe gegenüber den Männern (das ist nicht einmal tendenziell der Fall), und ebenso wenig die »Unwissenden« gegenüber den »Gebildeten« (wenn die Träger des Bewusstseins und der Organisation einer Klasse, „Unwissende« der modernen Gesellschaft, vom Schulsystem aufgegeben und von der bürgerlichen Kultur ausgeschlossen, sich faktisch zusammenschließen, geschieht dies bemerkenswerterweise im 
Namen der Wissenschaft und unter der Voraussetzung, in den eigenen Reihen oder außerhalb »organische Intellektuelle« zu finden).

27. Vgl. Jacques Rancière (2007).

28. Vgl. Olivier Schwartz (1990).

29. Der Unterschied der Geschlechter ist als soziale Teilung der Ersatz für die Transzendenz in der Gesellschaft, in der die Politik immanent ist (der postrevolutionären Gesellschaft), nachdem sie in der Gesellschaft, in der die Politik transzendent ist, eine der Metaphern der Macht war.

30. Die Erfahrung beweist, dass diese Triebkraft der nationalen Gemeinschaft in der sozialen Klassengemeinschaft ihr genaues Gegenstück hat: vgl. Françoise Duroux, La famille de l'ouvriermythe ou réalité (Diss., Universität Paris VII).

31. Dem widerspricht keineswegs die Berufsausbildung, insofern ihre Funktion wesentlich die Differenzierung in der Entintellektualisierung ist (dies schließt die Entintellektualisierung der Intellektuellen selbst, der Angestellten, ein).

INDEX

Mots-clés : droit naturel, droits de l'homme, droits du citoyen, égaliberté

Schlüsselwörter : Bürgerrechte, Gleichfreiheit, Menschenrechte, Naturrecht

\section{AUTEURS}

\section{ETIENNE BALIBAR}

Etienne Balibar ist emeritierter Professor an der Universität Paris X - Nanterre. Nähere Informationen finden Sie hier.

Gekürzte Fassungen dieses Artikels sind erschienen in Actuel Marx und Balibar (1992). Eine Übersetzung der Kurzfassung ist erschienen unter dem Titel »Menschenrechte ১Bürgerrechte`: Zur modernen Dialektik von Freiheit und Gleichheit«, in: Balibar, É. (1993): Die Grenzen der Demokratie, Hamburg, Argument, S. 99-123. 